ARTICLE

\title{
Adipocyte Piezo1 mediates obesogenic adipogenesis through the FGF1/FGFR1 signaling pathway in mice
}

\author{
ShengPeng Wang (10 1,2凶 , Shuang Cao ${ }^{1,2}$, Malika Arhatte ${ }^{3}$, Dahui $\mathrm{Li}^{4}$, Yue Shi ${ }^{2}$, Sabrina Kurz ${ }^{1}$, Jiong Hu (iD ${ }^{5}$, \\ Lei Wang ${ }^{1}$, Jingchen Shao ${ }^{1}$, Ann Atzberger ${ }^{6}$, Zheng Wang ${ }^{7}$, Changhe Wang ${ }^{8}$, Weijin Zang ${ }^{9}$, Ingrid Fleming ${ }^{5}$, \\ Nina Wettschureck ${ }^{1,10}$, Eric Honoré (i) ${ }^{3}$ \& Stefan Offermanns (i) ${ }^{1,10 凶}$
}

White adipose tissue (WAT) expansion in obesity occurs through enlargement of preexisting adipocytes (hypertrophy) and through formation of new adipocytes (adipogenesis). Adipogenesis results in WAT hyperplasia, smaller adipocytes and a metabolically more favourable form of obesity. How obesogenic WAT hyperplasia is induced remains, however, poorly understood. Here, we show that the mechanosensitive cationic channel Piezo1 mediates dietinduced adipogenesis. Mice lacking Piezo1 in mature adipocytes demonstrated defective differentiation of preadipocyte into mature adipocytes when fed a high fat diet (HFD) resulting in larger adipocytes, increased WAT inflammation and reduced insulin sensitivity. Opening of Piezo1 in mature adipocytes causes the release of the adipogenic fibroblast growth factor 1 (FGF1), which induces adipocyte precursor differentiation through activation of the FGF-receptor-1. These data identify a central feed-back mechanism by which mature adipocytes control adipogenesis during the development of obesity and suggest Piezo1mediated adipocyte mechano-signalling as a mechanism to modulate obesity and its metabolic consequences.

\footnotetext{
${ }^{1}$ Max Planck Institute for Heart and Lung Research, Department of Pharmacology, Ludwigstr. 43, 61231 Bad Nauheim, Germany. ${ }^{2}$ Cardiovascular Research Center, School of Basic Medical Sciences, Xi'an Jiaotong University Health Science Center, Key Laboratory of Environment and Genes Related to Diseases, No.76 West Yanta Road, Yanta District, Xi'an, China. ${ }^{3}$ Université Côte d'Azur, Centre National de la Recherche Scientifique, Institut de Pharmacologie Moléculaire et Cellulaire, Labex ICST, Valbonne, France. ${ }^{4}$ The State Key Laboratory of Pharmaceutical Biotechnology, Department of Pharmacology and Pharmacy, The University of Hong Kong, Hong Kong SAR, China. ${ }^{5}$ Institute for Vascular Signalling, Centre for Molecular Medicine, Goethe University, Frankfurt am Main, Germany. ${ }^{6}$ Max Planck Institute for Heart and Lung Research, Flow Cytometry Service Group, Ludwigstr. 43,61231 Bad Nauheim, Germany. ${ }^{7}$ Department of Hepatobiliary Surgery, First Affiliated Hospital of Xi'an Jiaotong University, Xi'an, China. ${ }^{8}$ Center for Mitochondrial Biology and Medicine, School of Life Science and Technology, Xi'an Jiaotong University, Xi'an, China. ${ }^{9}$ Department of Pharmacology, School of Basic Medical Sciences, Xi'an Jiaotong University Health Science Center, Xi'an, China. ${ }^{10}$ Center for Molecular Medicine, Goethe University Frankfurt, Theodor-Stern-Kai 7 , 60590 Frankfurt, Germany. ${ }^{凶}$ email: shengpeng.wang@xjtu.edu.cn; stefan.offermanns@mpi-bn.mpg.de
} 
T he white adipose tissue (WAT) is the primary site for fat storage and release. A dysbalance of this process leads to obesity and associated diseases including insulin resistance and type-2 diabetes, which affect a growing number of people worldwide ${ }^{1-4}$. Despite the essential role of WAT expansion in metabolic disorders, the mechanisms of WAT growth in obesity are still incompletely understood. Under increased caloric intake, WAT expands through an increase in the size of preexisting adipocytes (hypertrophy) as well as through the formation of new adipocytes from adipocyte precursor cells (adipogenesis) resulting in an increased number of adipocytes (hyperplasia) $)^{5-10}$. The balance of adipocyte hypertrophy and hyperplasia during obesity has a strong effect on the complications of obesity including type-2 diabetes. Whereas hypertrophy promotes adipose tissue inflammation and insulin resistance, hyperplasia results in smaller adipocytes and goes along with less adipose tissue inflammation and better insulin sensitivity 7,11 .

Adipogenesis under a hypercaloric diet is rapidly initiated by increased proliferation of adipocyte precursor cells, which are immature mesenchymal cells located in the stromal, vascularized tissue between adipocytes ${ }^{12-15}$. The subsequent differentiation of adipocyte precursor cells into mature adipocytes takes several weeks ${ }^{6,15}$. Multiple signaling pathways and mediators have been described which regulate pre-adipocyte proliferation and differentiation into mature adipocytes such as bone morphogenetic protein signaling and WNT signaling or insulin-like growth factor $1^{8,16-18}$. It is, however, incompletely understood through which mechanisms adipocyte progenitor cell proliferation and differentiation is controlled by systemic and local factors. It is also not clear, to what degree mature adipocytes sense a metabolic overload and functionally interact with progenitor cells to regulate adipogenesis.

Piezo1 and Piezo2 are homotrimeric mechanically activated non-selective cationic channels, which mediate mechanically induced currents ${ }^{19-21}$. Piezol has been shown to be gated directly by changes in membrane tension ${ }^{22-24}$ and to be involved in diverse processes including endothelial flow sensing ${ }^{25}$, osmotic homeostasis in erythrocytes ${ }^{26}$ or epithelial cell density regulation $^{27}$. Since we observed high expression of Piezol in mature white adipocytes we speculated that Piezo1-mediated mechanosignalling is involved in adipose tissue function and remodeling.

In this paper, we report that Piezol expressed in mature adipocytes is activated under a hypercaloric diet and induces adipocyte precursor differentiation through the release of the adipogenic mediator FGF1.

\section{Results}

Functional expression of Piezo1 in adipocytes. In a mouse line expressing $\beta$-galactosidase under the control of the Piezol promoter, we observed high expression in inguinal (subcutaneous) and epididymal (visceral) WAT (Fig. 1a). Quantitative RT-PCR and immunoblotting showed expression of Piezol, but not of Piezo2 in mature mouse and human adipocytes from visceral WAT (vWAT) and in differentiated 3T3-F442A adipocytes (Fig. $1 \mathrm{~b}$ and Supplementary Fig. 1a). Cell attached patch-clamp recordings of differentiated 3T3-F442A cells revealed the existence of large stretch-activated currents (Fig. 1c) with a reversal potential close to $0 \mathrm{mV}$, as expected for a non-selective cationic current, and with inactivation and deactivation kinetics remarkably slow compared to Piezo1 expressed in transfected HEK cells (Supplementary Fig. 1b, c). The knock-down of Piezol expression using validated siRNAs ${ }^{28}$ suppressed this stretch-activated current (Fig. 1c). Moreover, exposure of differentiated 3T3-F442A cells to the Piezol activator Yoda1 induced a transient increase in the free cytosolic $\mathrm{Ca}^{2+}$-concentration, which was not seen after
siRNA-mediated suppression of Piezo1 expression (Supplementary Fig. 1d). Similarly, in murine adipocytes, Yodal and also short term exposure to a hypotonic solution induced a significant increase in the free cytosolic $\mathrm{Ca}^{2+}$-concentration (Fig. 1d, e), an effect which was greatly reduced in adipocytes of tamoxifeninduced adipocyte-specific Piezo1-deficient mice (Ad-Piezo1-KO) (Fig. 1d, e). Since adipocytes show a significant increase in size already after 2 days of HFD feeding (Supplementary Fig. 1e, f), we used the fluorescent lipid tension sensor, Flipt $\mathrm{R}^{29}$ to determine membrane tension in mature adipocytes. We observed that adipocyte membrane tension significantly increases after 7 days of HFD feeding (Fig. 1f).

Loss of adipocyte Piezol promotes adipocyte hypertrophy under HFD. In Ad-Piezo1-KO mice, Piezol mRNA and protein were selectively suppressed in WAT upon induction of Cre by tamoxifen (Supplementary Fig. 1g, h) without eliciting changes in body weight, glucose tolerance and the size and number of adipocytes in the subcutaneous WAT (sWAT) and vWAT when on a standard diet (SD) (Supplementary Fig. 1i-l). When fed a HFD for 16 weeks, we observed a similar gain in body weight in control and Ad-Piezo1-KO littermates (Fig. 2a). Also the mass of sWAT, brown adipose tissue, and liver as well as the food intake, heat production, and respiratory quotient were not different between the two strains (Fig. 2b; Supplementary Fig. 2a-d). There was, however, a significant increase in vWAT mass of Ad-Piezo1-KO animals (Fig. 2b). Histological and flow cytometric analysis of the vWAT revealed an increased adipocyte sectional area and a pronounced increase in the number of large adipocytes (diameter $>110 \mu \mathrm{m}$ ) in Ad-Piezo1-KO animals (Fig. 2c-e and Supplementary Fig. 5a), whereas the total number of adipocytes in the vWAT was reduced (Fig. 2f). As described before ${ }^{6,15}$, HFD did not induce adipocyte hyperplasia in the sWAT (Supplementary Fig. 2e). A relative increase in adipocyte hypertrophy has been reported to result in increased insulin resistance and inflammation in humans and mice ${ }^{7,30-32}$. Consistent with this, Ad-Piezo1KO mice that received HFD exhibited a significantly reduced glucose tolerance (Fig. 2g), a modest increase in insulin resistance (Fig. 2h) and increased insulin plasma levels (Fig. 2i). The same animals had an increased numbers of $\mathrm{CD} 8^{+}$macrophages and higher levels of inflammatory marker genes in their vWAT (Fig. 2j, k) as well as increased IL-6 plasma levels (Fig. 2l).

Adipocyte Piezol is required for obesogenic adipogenesis. The reduced adipocyte number and increased adipocyte size in vWAT from HFD-fed Ad-Piezo1-KO mice suggested either an increase in adipocyte death or a decrease in HFD-induced adipogenesis, or a combination of both. Analysis of vWAT from Ad-Piezo1-KO mice revealed no sign of increased programmed cell death compared to wild-type animals such as increased caspase-3 activity, DNA fragmentation or crown-like structures (Fig. 3a-c and Supplementary Fig. 2f). Therefore, we focused on adipocyte hyperplasia in response to HFD feeding and performed adipocyte pulse-chase experiments ${ }^{15}$ in adipocyte-specific, tamoxifeninducible Cre transgenic mice (Adipoq-CreERT2) crossed with the $\mathrm{mT} / \mathrm{mG}$ Cre reporter line, which switches from membranetargeted Tomato expression to membrane-targeted GFP expression upon Cre-mediated recombination ${ }^{15}$ (Fig. 3d). Eight weeks after induction of Cre activity, $4 \%$ of the adipocytes in vWAT from wild-type and Ad-Piezo1-KO mice fed a SD were mTomato-positive (Fig. 3e and Supplementary Fig. 2g). In contrast, after 8 weeks of HFD vWAT from wild-type mice contained $\sim 20 \%$ mTomato-positive adipocytes, indicating that they formed from mTomato-positive adipocyte precursor cells after tamoxifen treatment (Fig. 3e, f). However, only half of the number of 
a

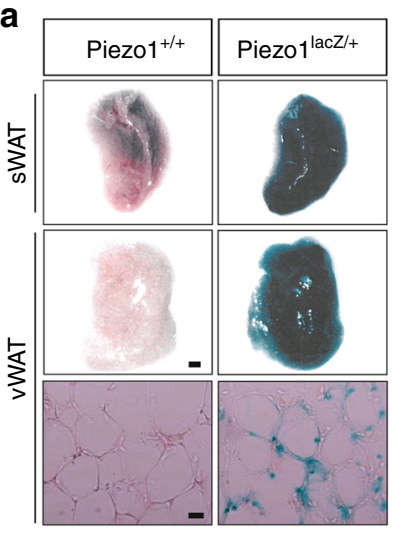

b

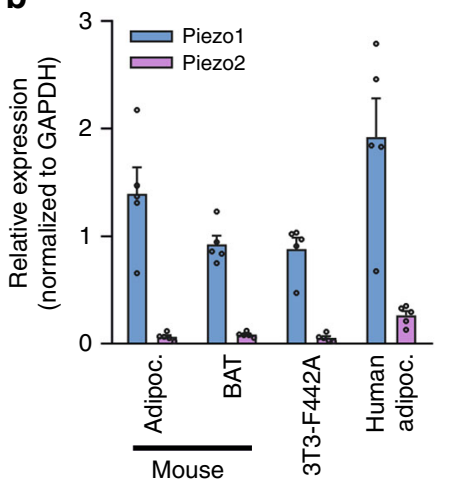

C

siRNA control siRNA Piezo1\#1 siRNA Piezo1\#2
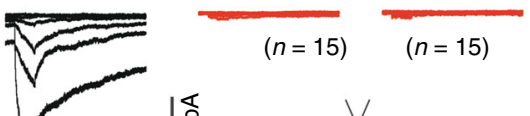

$(n=17) \quad \frac{⿱}{2}$

希 V

Cell-attached patch

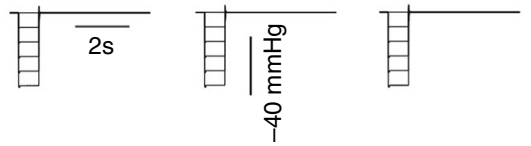

d

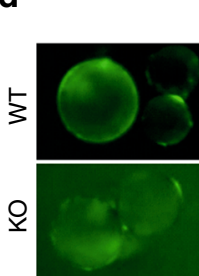

Os

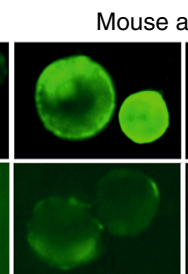

$30 \mathrm{~s}$

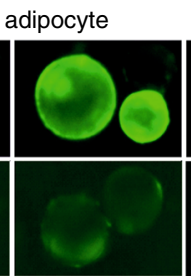

$60 \mathrm{~s}$

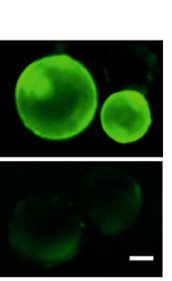

120 s
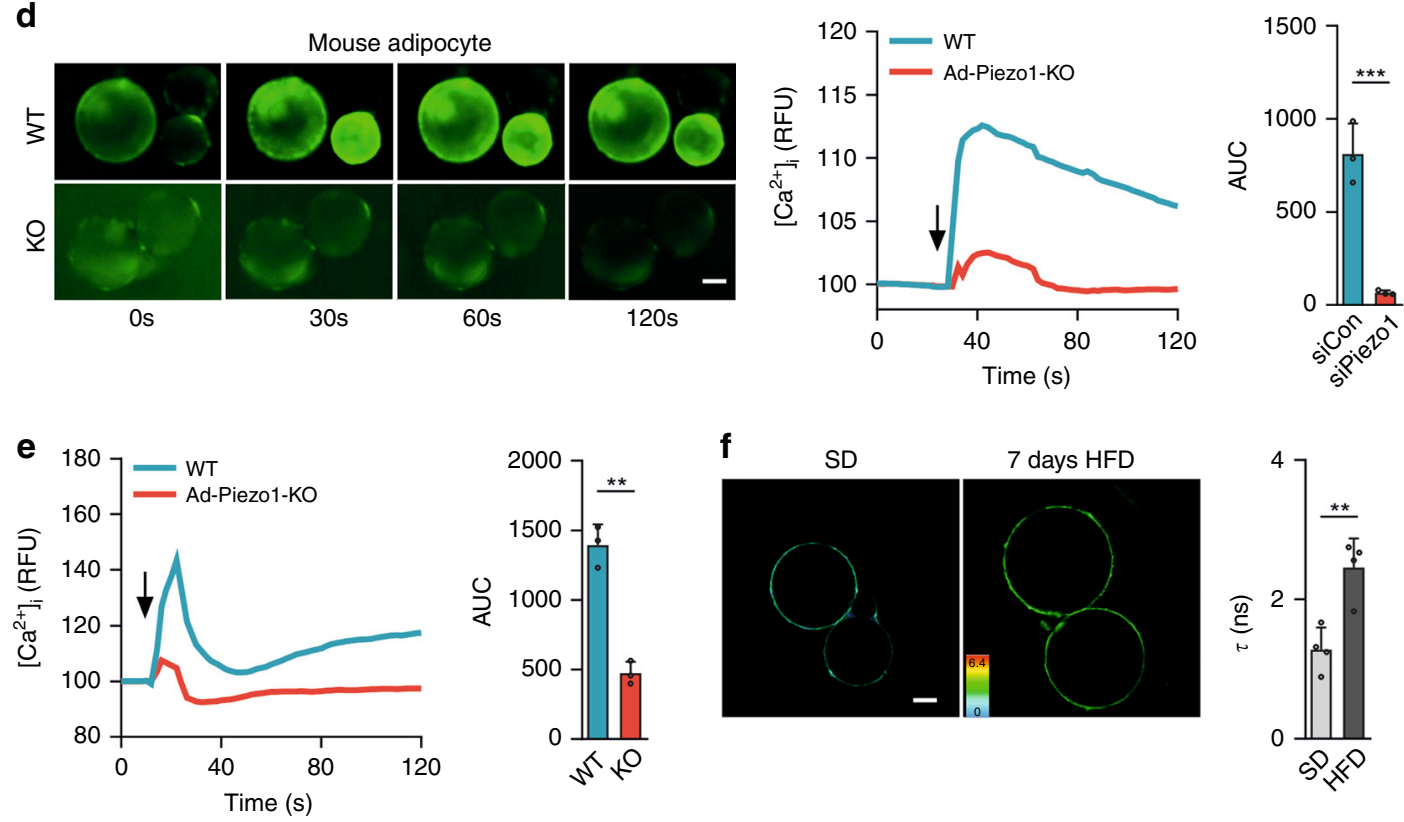

f

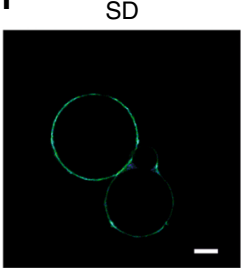

7 days HFD
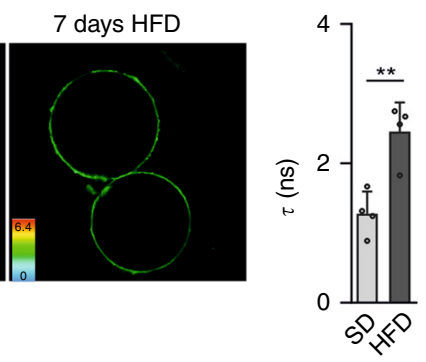

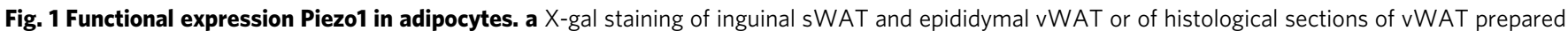

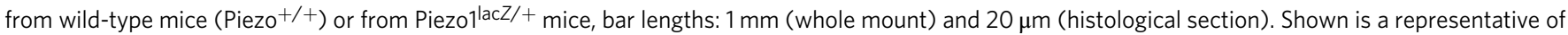
three independent experiments. b Expression of mRNAs encoding Piezo1 or Piezo2 in murine and human vWAT adipocytes, brown adipose tissue (BAT) and in differentiated 3T3-F442A adipocytes ( $n=5$ samples for each group). (c) Stretch-activated currents from differentiated 3T3-F442A were recorded in the cell-attached patch clamp configuration. The holding potential was $-80 \mathrm{mV}$ and the membrane was stretched by pulses of negative pressure with a

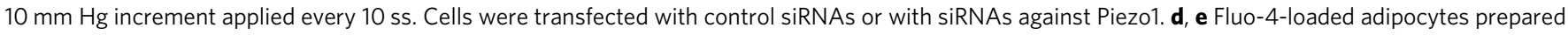
from vWAT of wild-type (WT) or Ad-Piezo1-KO mice were exposed to $10 \mu \mathrm{M}$ Yoda1 (d) or hypotonic buffer (210 mOsm/kg; e), and [Ca $\left.{ }^{2+}\right]_{i}$ was

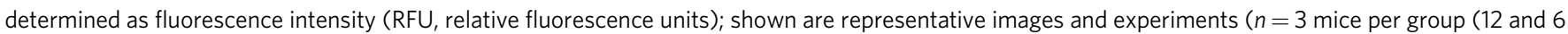
measurements per animal in $\mathbf{d}, \mathbf{e}$, respectively)). Arrows indicate the addition of Yoda1 or of hypotonic buffer. Bar diagrams show the area under the curve (AUC) of the $\mathrm{Ca}^{2+}$-transient. f Fluorescence lifetime $\tau_{1}$ images of FliptR in visceral adipocytes from wild-type mice kept under normal show or fed a HFD for 7 days. Corresponding lifetime mean values are shown in the bar diagram. The color bar corresponds to lifetime in nanoseconds ( $\mathrm{ns}$ ). $n=4$ mice per group (10 measurements per animal) (f). Bar lengths in d, f: $10 \mu \mathrm{m}$. Shown are mean values \pm s.e.m.; ${ }^{\star \star} P \leq 0.01$; ${ }^{\star \star \star} P \leq 0.001$ (two-tailed non-parametric Mann-Whitney $U$-test). Source data are provided as a Source data file.

mTomato-positive cells were found in Ad-Piezo1-KO mice (Fig. 3e, f). Thus, the recruitment of new adipocytes from adipocyte precursors was compromised in HFD-fed Ad-Piezo1-KO animals.

To test whether the defect in HFD-induced adipogenesis was due to reduced adipocyte precursor proliferation or differentiation, we labeled adipocyte precursors in WAT with bromodeoxyuridine (BrdU) or ethynyl-deoxyuridine (EdU) in vivo. After 1 week of HFD feeding, when increased adipocyte precursor proliferation was already observed ${ }^{15}$, the number of BrdU-labeled PDGFRa-positive vWAT stromal cells did not differ between wild-type and Ad-Piezo1-KO mice (Fig. 4a, b). Also, flow cytometric analysis of EdU incorporation into highly enriched
$\mathrm{Cd} 31^{-} ; \mathrm{Cd}_{4} 5^{-} ; \mathrm{PDGFRa}^{+}\left(\mathrm{Lin}^{-} ; \mathrm{PDGFR}^{+}\right)$adipocyte precursor cells showed no difference (Fig. $4 \mathrm{c}$ and Supplementary Fig. 5b). In contrast, after 8 weeks of HFD feeding, when BrdU-labeled adipocyte precursors differentiate into mature adipocytes ${ }^{15}$, the number of BrdU-positive adipocytes in vWAT of Ad-Piezo1-KO mice was decreased by $\sim 50 \%$ (Fig. $4 \mathrm{~d}$, e), a finding confirmed by flow cytometric analysis of EdU incorporation into adipocyte nuclei (Fig. 4f and Supplementary Fig. 5c). Numbers of EdUpositive $\mathrm{Lin}^{-}$;PDGFRa ${ }^{+}$precursor cells in the SVF on the other hand were increased in Ad-Piezo1-KO mice (Fig. $4 \mathrm{~g}$ and Supplementary Fig. 5b). We also found decreased expression of the early adipocyte differentiation markers Ppar 22 and C/ebpa in the SVF of HFD-fed Ad-Piezo1-KO mice (Fig. 4h). Thus, the loss 
a

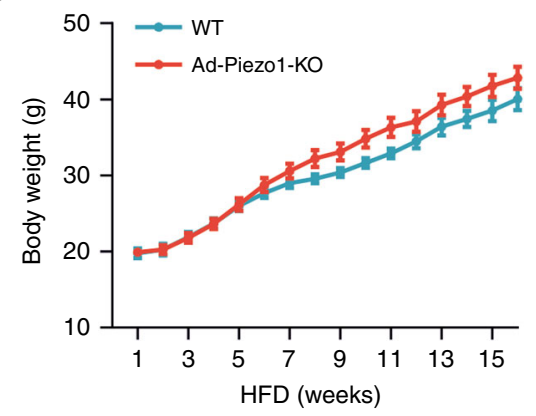

d

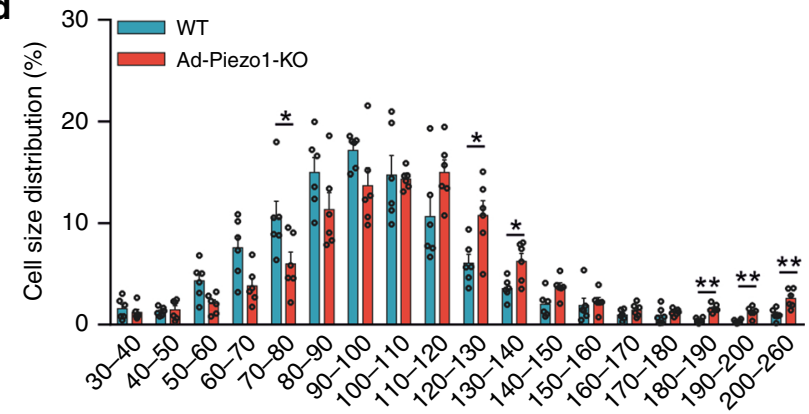

Diameter $(\mu \mathrm{m})$

h
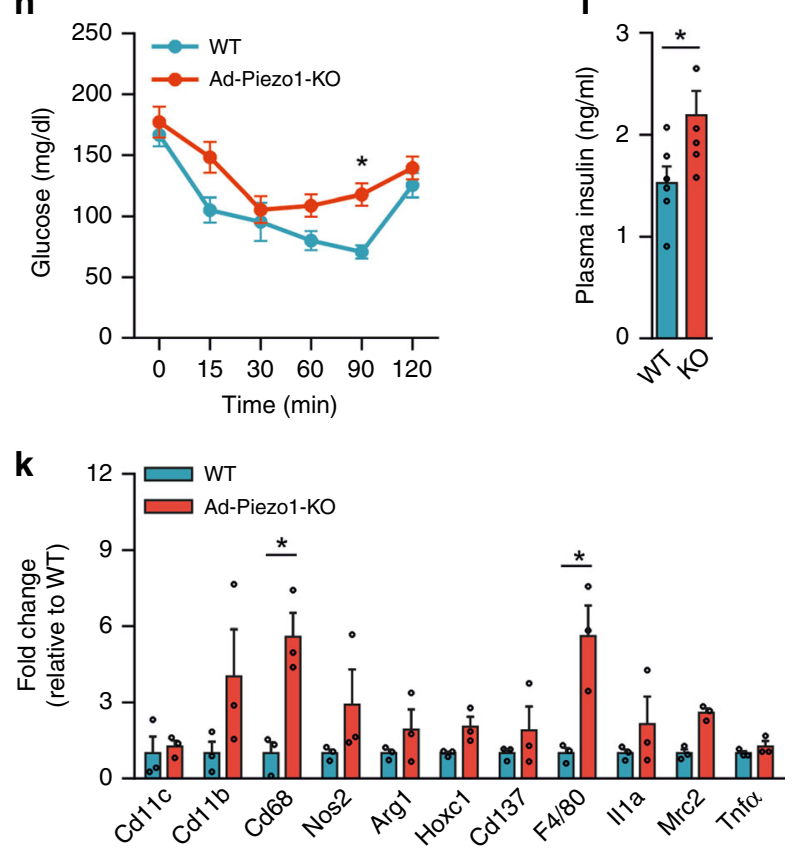

i

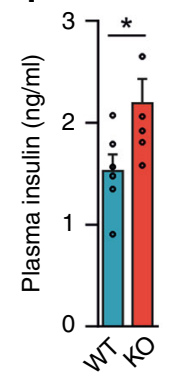

b

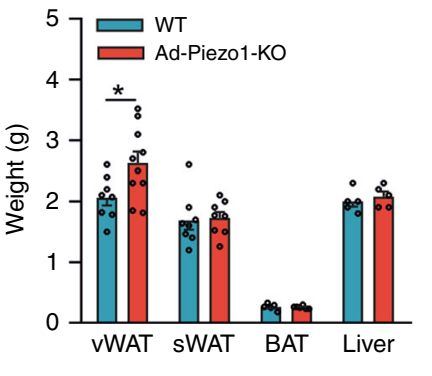

e

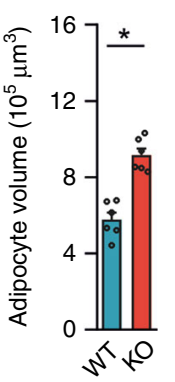

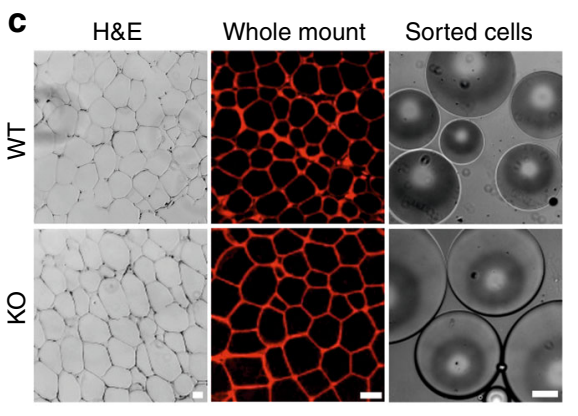

$\mathbf{f}$

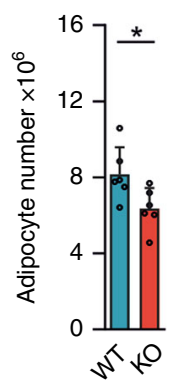

g

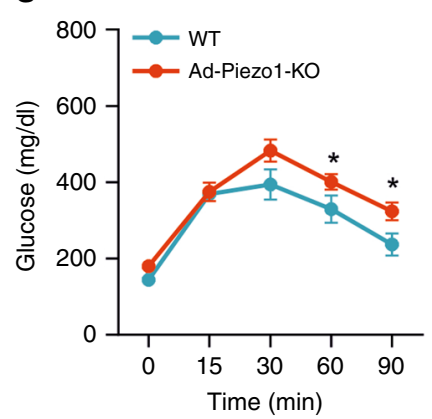

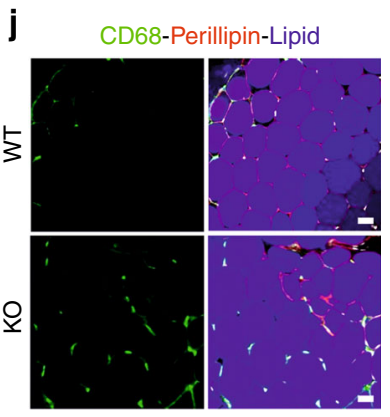

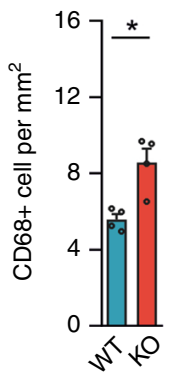

Fig. 2 Metabolic phenotype of Ad-Piezo1-KO mice under HFD. a Body weight of wild-type (WT) or Ad-Piezo1-KO mice during HFD feeding for 16 weeks $(n=11$ mice $(W T), n=12$ mice $(K O)$ ). $\mathbf{b}$ Weight of epididymal vWAT, inguinal sWAT, BAT and the liver from wild-type (WT) or Ad-Piezo1-KO mice fed a HFD for 16 weeks ( $n=8$ mice ( $v W A T$ WT and sWAT); $n=5$ mice (BAT WT and liver); $n=10$ mice ( $v W A T$ KO); $n=6$ mice (BAT KO)). c H\&E-stained vWAT sections, whole-mount vWAT stained with anti-perilipin antibodies and sorted vWAT adipocytes from wild-type (WT) and Ad-Piezo1-KO (KO) mice; bar length: $50 \mu \mathrm{m}$. Shown is a representative of 4 independent experiments. d Distribution of size of vWAT adipocytes prepared from wild-type (WT) and Ad-Piezo1-KO mice fed HFD for 16 weeks ( $n=6$ mice (WT and KO each)). e, $\mathbf{f}$ Average volume (e) and total number (f) of adipocytes in epididymal vWAT from wild-type (WT) and Ad-Piezo1-KO (KO) mice ( $n=6$ mice (WT and KO each)). g-i Glucose tolerance (g), insulin tolerance (h) and plasma insulin levels (i) in wild-type (WT) and Ad-Piezo1-KO mice (KO) fed a HFD for 16 weeks ( $n=6$ mice (WT in $\mathbf{g}$, WT and KO in $\mathbf{i}, \mathbf{h}$ ), $n=7$ mice (KO in $\mathbf{g}$ )). $\mathbf{j}$ Whole mount of vWAT from wild-type (WT) or Ad-Piezo1-KO mice (KO) stained with anti-CD68 and anti-perilipin antibodies as well as with LipidTOX (lipid); bar length: $50 \mu \mathrm{m}$. The bar diagram shows the statistical evaluation of CD68-positive cells per area $(n=4$ mice (at least 10 sections per mouse)). k, I Expression of inflammation marker genes in VWAT (k) and IL-6 levels in plasma (I) from wild-type (WT) and Ad-Piezo1-KO mice (KO) fed HFD for 16 weeks ( $n=3$ mice (WT and KO each in $\mathbf{k}$ ), $n=6$ mice (WT and KO each in I)). Shown are mean values \pm s.e.m.; ${ }^{\star} P \leq 0.05$; ${ }^{\star \star} P \leq 0.01 ; n . s .$, not significant (two-tailed non-parametric Mann-Whitney U-test). Source data are provided as a Source data file. 

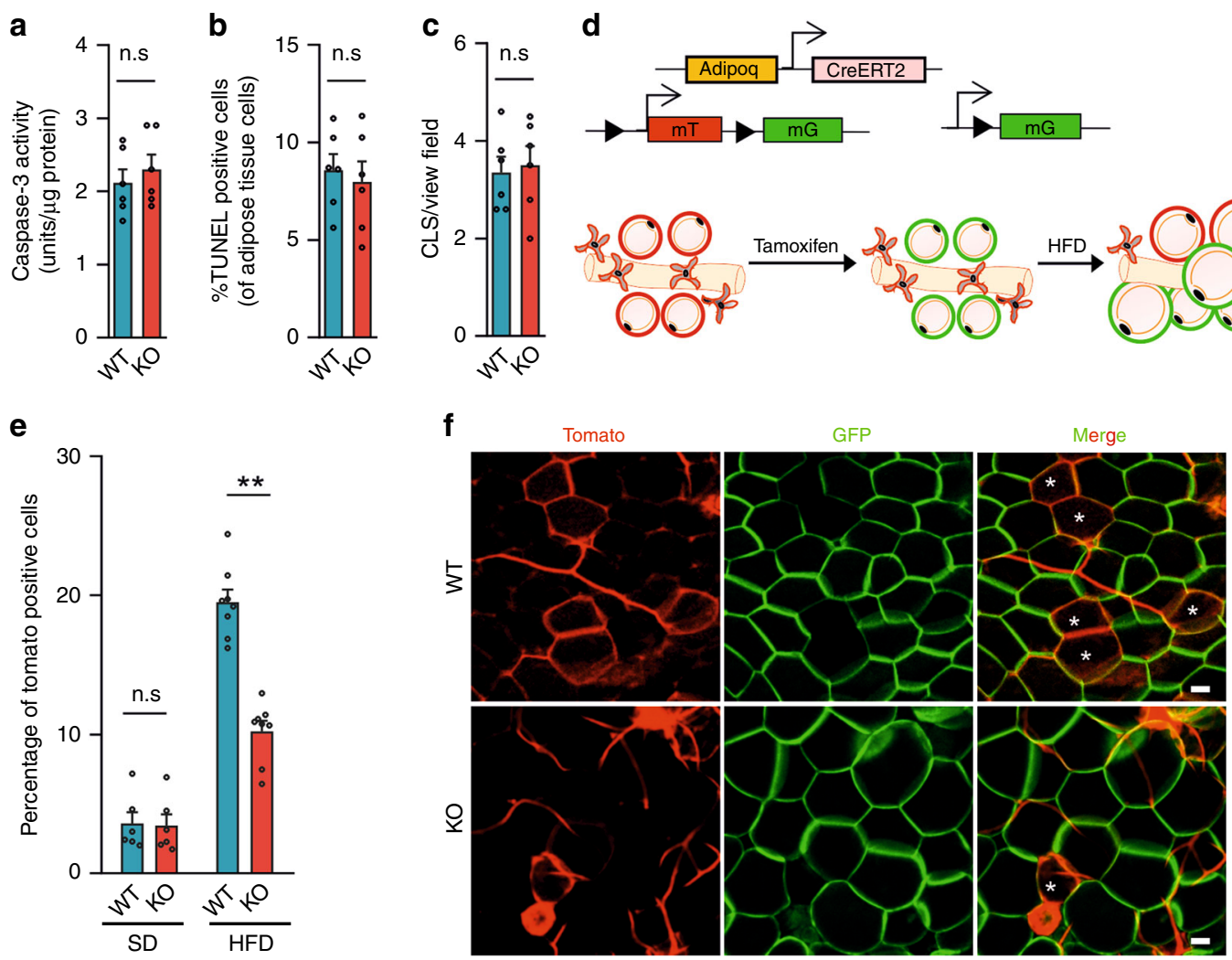

Fig. 3 Analysis of in vivo adipogenesis in Ad-Piezo1-KO mice. a-c Caspase-3 activity (a), TUNEL staining (b) and crown-like structures (CLS; c) in vWAT from wild-type (WT) and Ad-Piezo1-KO mice (KO) fed HFD for 16 weeks ( $n=6$ mice (at least 10 sections per mouse in $\mathbf{b}, \mathbf{c})$ ). $\mathbf{d}$ Experimental design of analysis of adipogenesis in vivo using Adipoq-CreERT2;mT/mG mice. e, $\mathbf{f}$ Statistical analysis (at least 20 sections per mouse; e) and representative images (f) of adipocyte tracing in Adipoq-CreERT2;mT/mG;Piezo1 flox/flox (KO) and Adipoq-CreERT2;mT/mG;Piezo1 ${ }^{+/+}$mice (WT) after tamoxifen treatment and 8 weeks of HFD or SD in vWAT ( $n=6$ mice (SD), $n=8$ mice (HFD)); bar length: $50 \mu$ m. Shown are mean values \pm s.e.m.; ${ }^{\star \star} P \leq 0.01 ; n . s .$, not significant (two-tailed non-parametric Mann-Whitney $U$-test). Source data are provided as a Source data file.

of Piezol in mature adipocytes has no effect on adipocyte precursor proliferation, but strongly affected their differentiation into new adipocytes.

Piezo1 induces adipocyte precursor differentiation via FGF1. Consistent with the in vivo observation, we found no difference between conditioned medium from mature adipocytes isolated from HFD-fed wild-type and Ad-Piezo1-KO mice with regard to its effect on proliferation of 3T3-F442A preadipocytes or SVF cells (Fig. 5a). In contrast, conditioned medium from adipocytes from wild-type HFD-fed animals induced the differentiation of preadipocytes and SVF cells, whereas conditioned medium from Piezo1-deficient adipocytes or from adipocytes obtained from SD-fed mice had little effect on differentiation (Fig. 5a-c). This suggested that adipocytes from HFD-fed mice produce a transferrable factor in a Piezo1-dependent manner, that promotes the differentiation of adipocyte precursor cells. Several known adipogenic mediators including IGF1, FGF1, FGF2, Jag-1, TGF $\beta$, adiponectin, BMP3, and WNT5a ${ }^{17,18,32}$ (Fig. 6a) as well as several lipids (Supplementary Table 1) were found to be released from mature wild-type adipocytes, but only the release of FGF1 was increased by the Piezo1 activator Yoda1 (Fig. 6a, and Supplementary Table 1), and FGF1 release was also increased by hypoosmotic membrane stress induced by short term exposure to a hypotonic solution in mouse and human adipocytes (Fig. 6b, c). Consistent with reports that FGF1 is released from cells in a nonclassical manner by direct translocation through the plasma membrane ${ }^{33}$, Piezo1-dependent FGF1 release from adipocytes was not affected by the vesicle formation and transport inhibitor brefeldin A, but was blocked by chelation of intracellular $\mathrm{Ca}^{2+}$ using BAPTA-AM (Fig. 6d, e). In Piezo1-deficient adipocytes, FGF1 expression was unchanged (Supplementary Fig. 3a-c), but the basal FGF1 release, as well as the effect of Yodal and hypoosmotic membrane stress on FGF1 release, was clearly reduced (Fig. 6a, b and Supplementary Table 1). The ability of conditioned medium to induce preadipocyte differentiation was strongly decreased after depletion of FGF1 from the medium (Supplementary Fig. 2d, e), and the defect of conditioned medium from Piezol-deficient adipocytes to induce preadipocyte differentiation was rescued by addition of FGF1 as indicated by an increased number of lipid-containing cells while the average lipid content per cell remained unchanged (Fig. 6f and Supplementary Fig. 3f, g). Antagonism of FGF receptor 1 (Fgfr1), the major FGF receptor expressed in preadipocytes (Supplementary Fig. 3h, i), with PD173074 significantly reduced preadipocyte differentiation in response to FGF1 and conditioned medium from mature wildtype adipocytes (Fig. 6f). To further test the involvement of FGFR1 in the regulation of preadipocyte differentiation we generated mice lacking FGFR1 in preadipocytes by crossing mice carrying a floxed Fgfr1 allele $^{34}$ with Pdgfra-CreERT2 animals ${ }^{35-37}$ (Pa-Fgfr1-KO; Supplementary Fig. 4a, b). Loss of FGFR1 in adipocyte precursor cells of the SVF strongly inhibited the ability of conditioned medium (CM) of adipocytes prepared from HFD-fed wild-type mice to induce adipocyte differentiation as indicated by strongly reduced lipid droplet formation (Fig. 6g). The SVF from induced PdgfraCreERT2; Fgfr 1 flox/flox also did not show an increase the expression of several adipocyte marker genes including Ppar 2 and C/ebpa when exposed to adipocyte CM from HFD fed mice 
a

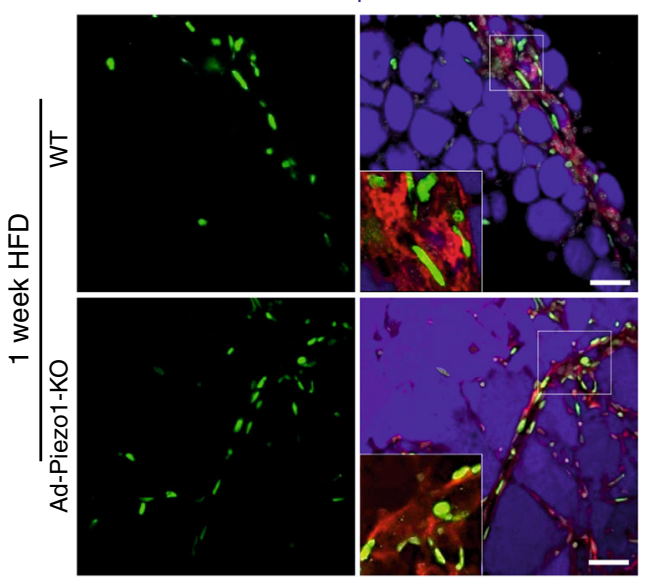

b

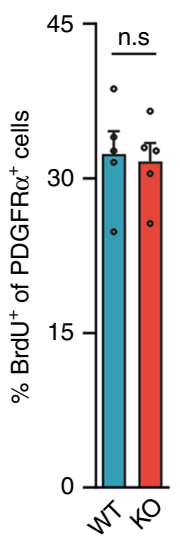

C

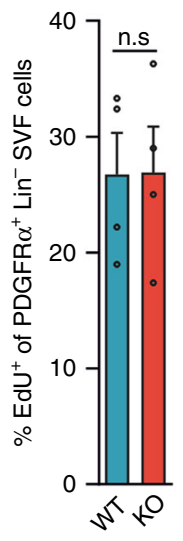

d

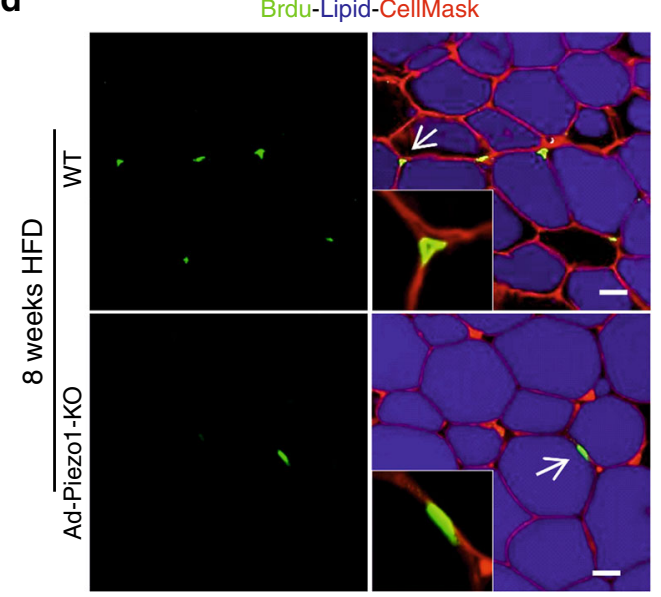

e

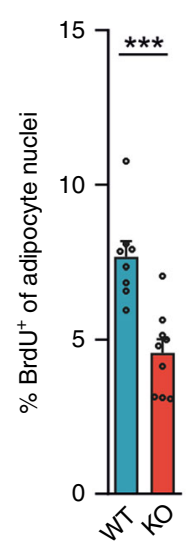

f

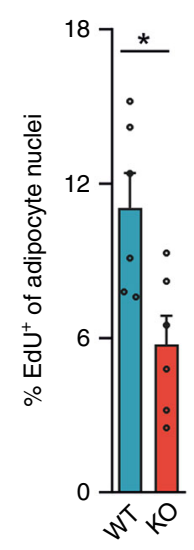

g

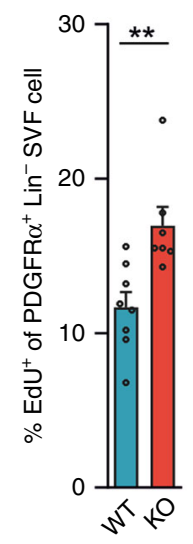

h

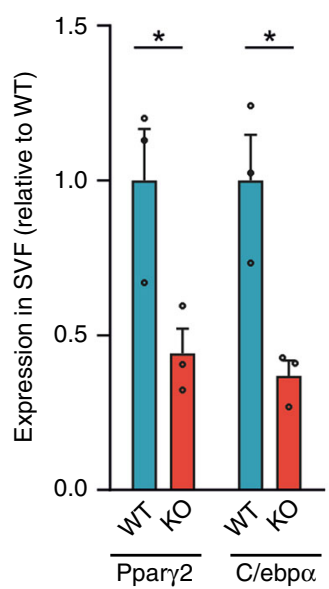

Fig. 4 Analysis of adipocyte precursor proliferation and differentiation in Ad-Piezo1-KO mice. a-g Wild-type (WT) and Ad-Piezo1-KO mice (KO) received $\operatorname{BrdU}(\mathbf{a}, \mathbf{b}, \mathbf{d}, \mathbf{e})$ or EdU $(\mathbf{c}, \mathbf{f}, \mathbf{g})$ for 1 week while feeding HFD for 1 week $(\mathbf{a}-\mathbf{c})$ or 8 weeks $(\mathbf{d}-\mathbf{g})$ started. Shown are representative images $(\mathbf{a}$, d) and quantifications ( $\mathbf{b}, \mathbf{e}$ ) of immunofluorescence staining for BrdU in nuclei of SVF stained with an anti-PDGFR $\alpha$ antibody and LipidTOX (lipid) (a, $\mathbf{b}$ ) or in adipocyte nuclei of VWAT (d, e). Tissues were stained with LipidTOX (lipid) and CellMask to visualize adipocyte plasma membranes. Insets show magnifications of boxed areas (d) or of typical adipocyte nuclei indicated by arrows (d); bar length: $50 \mu \mathrm{m}$ (a) and $20 \mu \mathrm{m}$ (d) ( $n=5$ mice (at least 12 sections per mouse in $\mathbf{b}$ ), $n=8$ or 9 mice (WT or $\mathrm{KO}$ in $\mathbf{e}$; at least 15 sections per mouse)). $\mathbf{c}, \mathbf{f}, \mathbf{g}$ Flow-cytometric analysis of EdU incorporation in PDGFR $\alpha$-positive, CD31-negative and CD45-negative (Lin-;PDGFR $\alpha^{+}$) cells of the vWAT SVF (c, $\mathbf{g}$ ) or in adipocyte nuclei (f) of wild-type (WT) and AdPiezo1-KO mice (KO) ( $n=4$ mice (WT and KO in $\mathbf{c}$ ), $n=6$ mice (WT and $K O$ in $\mathbf{f}), n=8$ mice (WT in $\mathbf{g}$ ), $n=7$ mice (KO in $\mathbf{g}$ )). In $\mathbf{c}, \mathbf{f}, \mathbf{g}$ at least 5000 cells were analyzed per animal. $\mathbf{h}$ Expression of mRNA encoding Ppary2 and C/ebp $\alpha$ in the SVF of vWAT prepared from wild-type (WT) and Ad-Piezo-KO mice (KO) fed a HFD for 16 weeks ( $n=3$ mice (WT and KO in h). Shown are mean values \pm s.e.m.; ${ }^{\star} P \leq 0.05$; ${ }^{\star \star} P \leq 0.01$; ${ }^{\star \star \star} P \leq 0.001$; n.s., not significant (two-tailed non-parametric Mann-Whitney U-test). Source data are provided as a Source data file.

or to FGF1 (Supplementary Fig. 4c, d). The FGF1-induced differentiation of SVF cells was reduced by the phosphatidylinositol 3-kinase (PI-3-K) inhibitor LY294002 as well as by the MAPkinase kinase (MEK1/MEK2) inhibitor PD98059, whereas the p38 mitogen-activated protein kinase inhibitor SB253580 had no effect, and a combination of the PI-3-kinase and p38 kinase inhibitor blocked the effect of FGF1 (Supplementary Fig. 4d). Induced Pdgfra-CreERT2;Fgfr $1^{\text {flox/flox }}$ mice fed a HFD recapitulated the phenotype of Ad-Piezo1-KO animals with increased adipocyte size and reduced adipocyte number (Fig. 6h-j and Supplementary Fig. 4e, f). When we in vivo-labeled proliferating adipocyte precursors in WAT of induced Pdgfra-CreERT2; Fgfr 1 flox/flox mice with BrdU during the first week of HFD feeding, we found after 8 weeks a reduced differentiation of adipocyte precursors into mature adipocytes as indicated by the strongly reduced number of BrdU-positive adipocytes (Fig. 6k). In contrast, the number of BrdU-labeled PDGFRa-positive vWAT stromal cells did not differ between wild-type and Pdgfra-CreERT2; Fgfr $1^{\text {flox/flox }}$ mice after 1 week (Supplementary Fig. 4g).

\section{Discussion}

The fact that loss of Piezol in mature adipocytes results in a reduced differentiation of adipocyte precursor cells under a HFD indicates that mature adipocytes generate an adipogenic signal which promotes adipocyte precursor differentiation. Among several candidates, we identified FGF1 as a mediator which is released from adipocytes upon Piezol activation and promoted adipocyte precursor differentiation. In line with our findings, FGF1 expression has been shown to increase in obese humans and mice ming $^{32,38}$ and to promote preadipocyte differentiation ${ }^{39,40}$. The role of FGF1 as a mediator of Piezo1-dependent adipogenesis is also supported by the fact that the phenotype of adipocytespecific Piezo1-deficient animals resembles that of FGF1-deficient 
a

3T3-F442A

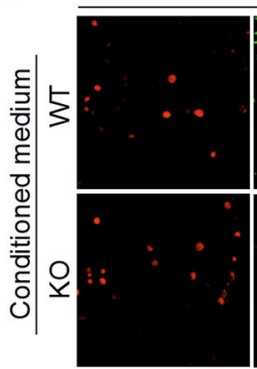

\section{(1)}

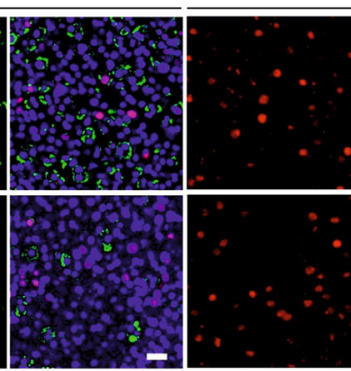

Ki67-Lipid-DAPI

b

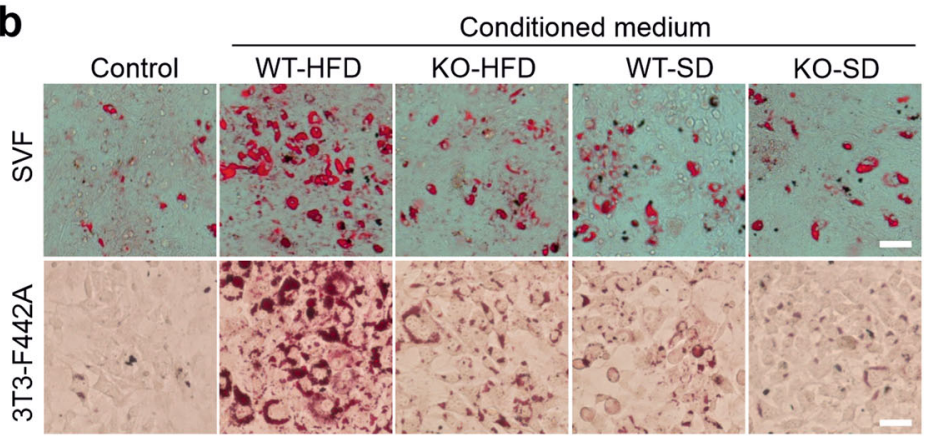

SVF
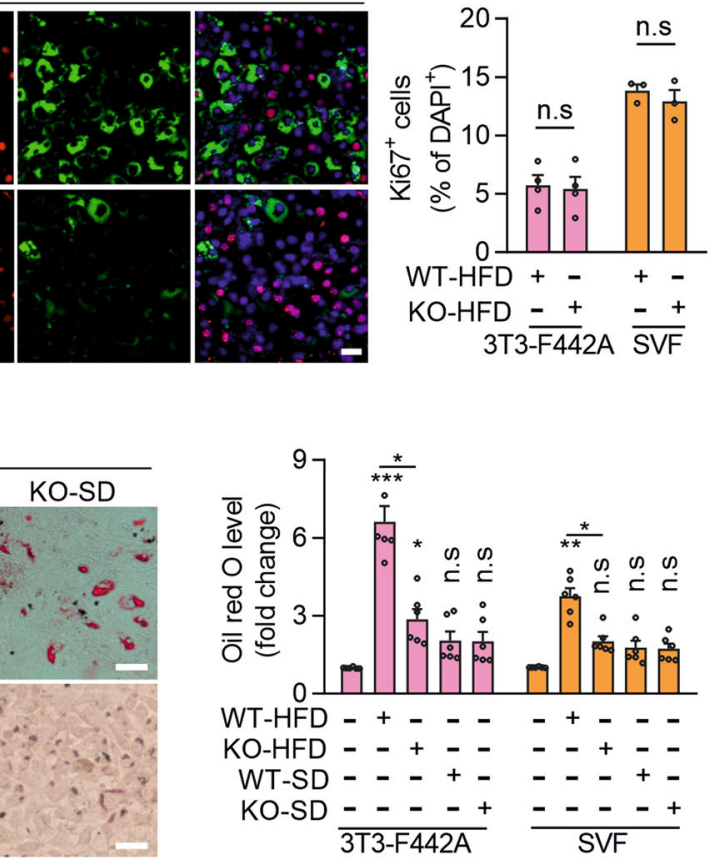

C

3T3-F442A

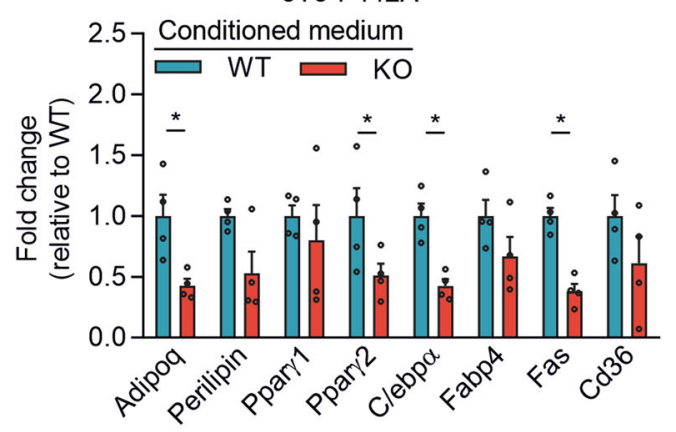

Fig. 5 Effect of conditioned medium from vWAT adipocytes on preadipocyte proliferation and differentiation. a-c Effects of conditioned medium from vWAT adipocytes prepared from wild-type (WT) and Ad-Piezo1-KO (KO) mice fed a HFD (a-c) or SD (b) for 16 weeks or of unconditioned medium (control in b). In a, proliferation of 3T3-F442A cells or of cells of the SVF was visualized by staining for Ki67, and cells were counterstained with DAPI and BODIPY (lipid). Shown are representative images and the statistical evaluation ( $n=4$ mice (WT and KO, 3T3-F442A), $n=3$ mice (WT and KO, SVF); at least 10 fields were analyzed per mouse). Bar length: $50 \mu \mathrm{m}$. In $\mathbf{b}$, SVF cells or undifferentiated 3T3-F442A cells were exposed to the medium and stained with Oil-red-O. Shown are representative images and the statistical evaluation of Oil-red-O content ( $n=6$ mice (for all groups)); bar length: $50 \mu \mathrm{m}$. In c, expression of different adipocyte marker genes in 3T3-F442A cells ( $n=4$ mice (for all groups)) was determined. Shown are mean values \pm s.e.m.; ${ }^{\star} P \leq$ $0.05 ;{ }^{\star \star} P \leq 0.01$; ${ }^{\star \star \star} P \leq 0.001$; n.s., not significant (compared to control in b) (two-tailed non-parametric Mann-Whitney $U$-test). Source data are provided as a Source data file.

mice, which upon feeding with HFD show an increased number of very large adipocyte, decreased insulin sensitivity, increased inflammation and a restriction of the phenotype to the visceral adipose tissue $^{32}$. We also identified FGFR1 as the FGF-receptor subtype on preadipocytes which mediates the effect of FGF1. Our in vitro data indicate that this FGFR1-mediated effect involved PI-3-kinae/AKT and MEK/ERK signaling. Interestingly, in Pdgfra-Cre;Akt2 flox/flox mice, loss of AKT2 in adipocyte precursor cells leads to reduced HFD-induced adipogenesis ${ }^{15}$ similar to the phenotype we observed in induced Pdgfra-CreERT2; Fgfr $1^{\text {flox/flox }}$ animals. This suggests that FGFR1 and AKT2 may function in the same pathway in preadipocytes.

Piezol can be activated by various mechanical stimuli acting on cellular membranes including mechanical indentation of the cell surface, cell migration, exposure to fluid shear stress, compression of the cell membrane, osmotic swelling or forces generated at the cell-cell or cell-matrix interface ${ }^{41,42}$. How Piezol is activated in hypertrophic adipocytes is not known. According to the law of Laplace, it is conceivable that the increase in adipocyte volume per se might result in an increase in plasma membrane tension sufficient to activate Piezol. In fact, we show that membrane tension in adipocytes increases already after 7 days of HFD feeding. In addition, compressive forces or strain exerted by the extracellular matrix on expanding adipocytes have been described ${ }^{43,44}$ and may also be able to induce Piezol activation. Strikingly, native adipocyte Piezol currents lacked the classical fast inactivation ${ }^{28}$, a phenomenon described in other cells before ${ }^{45}$. While the mechanism of slow inactivation kinetics remains unknown, it indicates that adipocyte Piezol might operate in response to chronic mechanical stress as expected for hypertrophic adipocytes.

Recently, evidence was provided that the volume-regulated anion channel (VRAC) is activated by an increase in adipocyte 
a

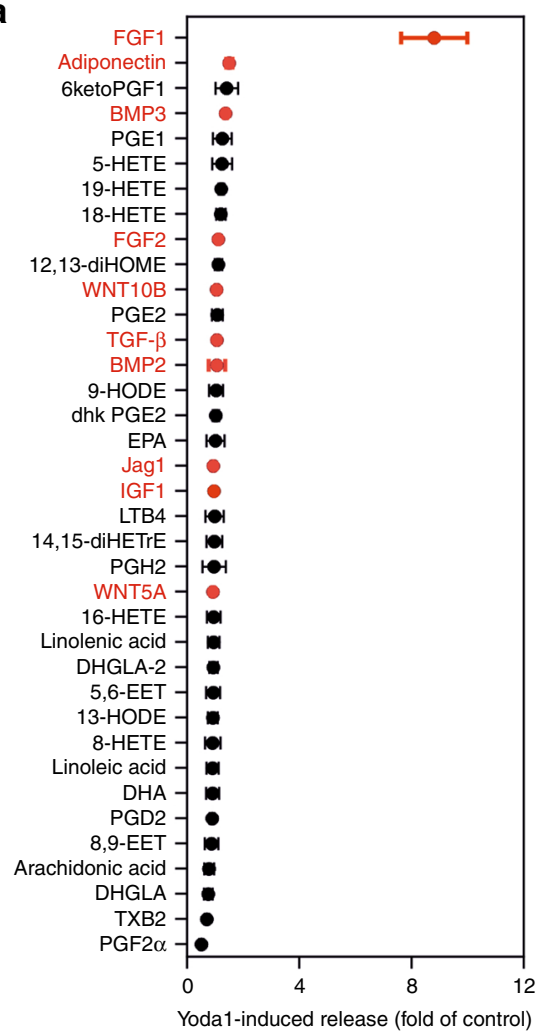

b

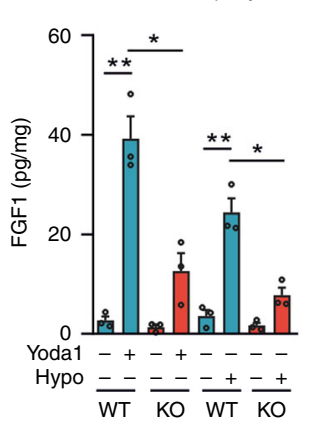

C

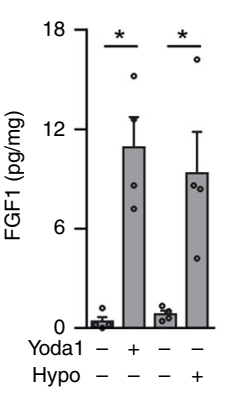

d

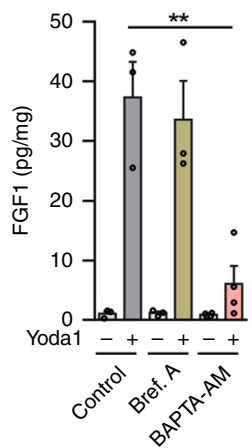

e

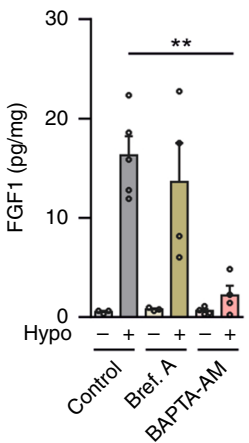

f

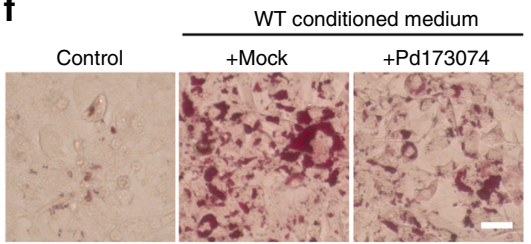

Ad-Piezo1-KO conditioned medium
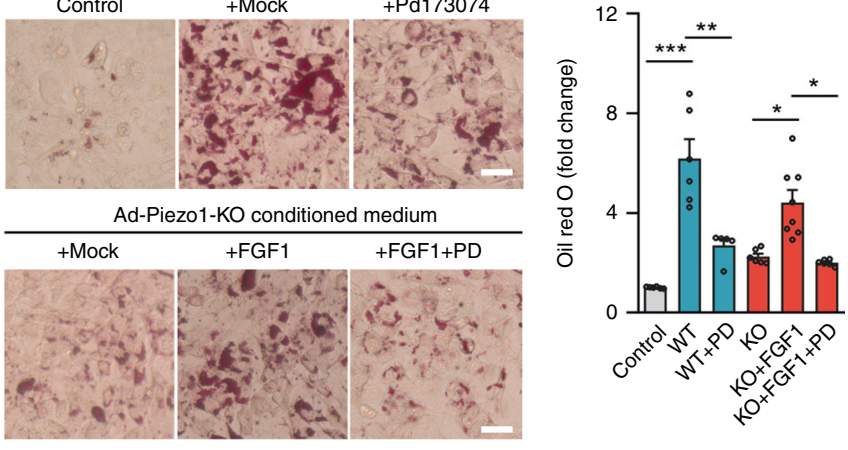

g

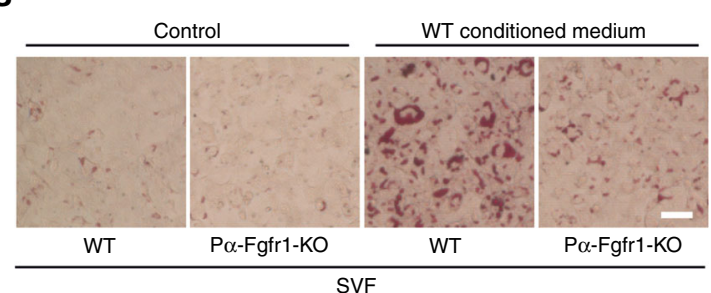

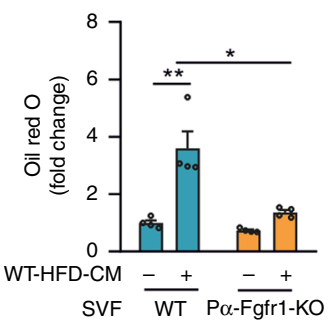

h

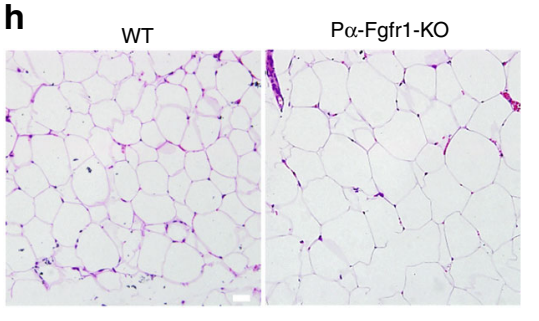

i

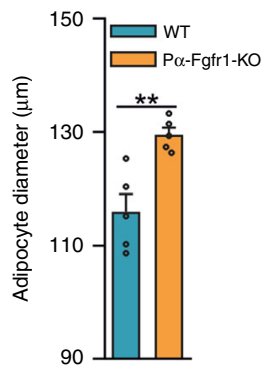

j

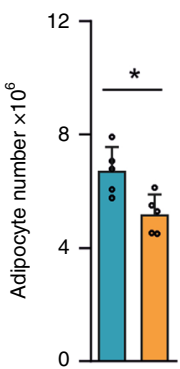

k

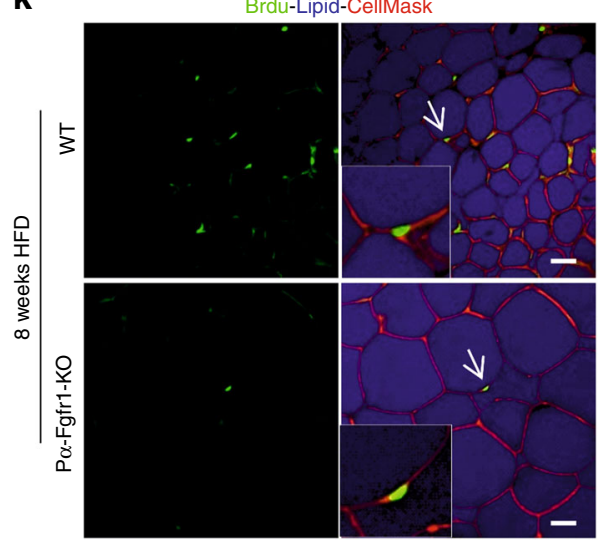

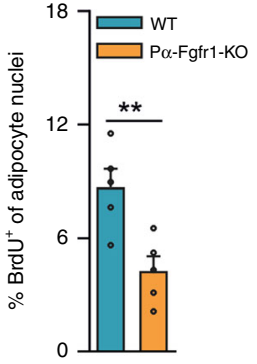

volume during adipocyte hypertrophy ${ }^{46}$. In contrast to the role of Piezo1 in visceral adipocytes described here, VRAC activation rather promotes adipocyte expansion and insulin signaling, and loss of VRAC results in reduced adipocyte size under HFD ${ }^{46}$. While VRAC thereby mediates a feed-forward mechanism to promote adipocyte expansion and to enhance insulin signaling during increased caloric intake, Piezol rather functions as a feedback regulator which promotes adipogenesis under a hypercaloric diet via FGF1 and FGFR1. Thus, VRAC and Piezo1 both appear to be part of a complex regulatory system by which mature adipocytes control WAT homeostasis during obesity.

A consequence of induced adipocyte-specific loss of Piezo1 expression was a reduced glucose tolerance and insulin sensitivity as well as an increased inflammatory activity in the visceral adipose tissue under HFD, a phenotype also seen in constitutive adipocyte-specific Piezo1-deficient mice ${ }^{47}$. In contrast to our findings in acutely induced adipocyte-specific Piezo1-deficient mice, constitutive Piezol-deficient mice had no significantly 
Fig. 6 FGF1 mediates Piezo1-dependent regulation of adipocyte differentiation. a Yoda1 $(10 \mu M)$ effect on release of protein mediators (red) and lipids (black) from mouse adipocytes (ratio of release in the presence and absence of Yoda-1 $(n=5$ mice)). b, $\mathbf{c}$ Effect of Yoda1 (10 $\mu M$ ) or hypotonic buffer (hypo; $210 \mathrm{mOsm} / \mathrm{kg}$ ) on FGF1 release from vWAT adipocytes from wild-type (WT) and Ad-Piezo1-KO mice (KO) (b) or from human vWAT (c) ( $n=3$ mice (b), $n=4$ samples (c)). d, e Effect of Yoda1 $(10 \mu \mathrm{M}$; d) or hypotonic buffer (hypo; $210 \mathrm{mOsm} / \mathrm{kg} ; \mathbf{e}$ ) on FGF1 release from mouse adipocytes pretreated in the absence or presence of brefeldin A (bref. A) or BAPTA-AM ( $n=3$ mice (control and bref. A in $\mathbf{d}$ as well as control and control + bref. A in e) $; n=5$ (control + hypo in e) $n=4$ mice (BAPTA in $\mathbf{d}$ and all other groups)). $\mathbf{f}$ PD173074 (PD; $10 \mu \mathrm{M}$ ) effect on 3T3-F442A cell differentiation induced by conditioned medium of vWAT adipocytes from wild-type mice fed HFD for 16 weeks and effect of FGF1 (10 ng/ml) and FGF1 + PD173074 on differentiation of 3T3-F442A cells exposed to conditioned medium from vWAT adipocytes from KO animals. Control: non-conditioned medium. Cells were stained with Oil-red-O. $\mathbf{g}$ Differentiation of SVF cells from wild-type or Pdgfr $\alpha$-CreERT2;Fgfr1 flox/flox (P $\alpha$-Fgfr1-KO) mice exposed to conditioned medium from wild-type adipocytes from HFD-fed mice (Oil-red-O staining; $n=4$ mice per condition). $\mathbf{h} \mathrm{H \& E}$-stained vWAT sections from wild-type (WT) and P $\alpha$ Fgfr1-KO mice fed a HFD for 12 weeks (one representative of three independent experiments). $\mathbf{i}, \mathbf{j}$ Average diameter (i) and total number (j) of adipocytes in vWAT from wild-type (WT) and P $\alpha$-Fgfr1-KO mice fed a HFD ( $n=5$ mice each). $\mathbf{k}$ Wild-type (WT) and P $\alpha$-Fgfr1-KO mice received BrdU during the first week of an 8-week HFD feeding period. Representative images and quantifications of immunofluorescence staining for BrdU in adipocyte nuclei of vWAT after HFD feeding $\left(n=5\right.$ mice ( $\geq 15$ sections per mouse)). Bar lengths in $\mathbf{f}, \mathbf{g}, \mathbf{h}, \mathbf{k}: 50 \mu \mathrm{m}$. Shown are mean values \pm s.e.m.; ${ }^{\star} P \leq 0.05 ;{ }^{\star \star} P \leq 0.01 ;{ }^{\star \star \star} P \leq$ 0.001; n.s., not significant (two-tailed non-parametric Mann-Whitney U-test). Source data are provided as a Source data file.

reduced adipocyte size but showed an increase in liver triglyceride content ${ }^{47}$. This may be the consequence of compensatory changes induced by long-term loss of Piezol in the constitutive knock-out model. A reduction in glucose tolerance and insulin sensitivity as well as increased adipose tissue inflammation were also observed in other mouse models with increased adipocyte cell size and decreased adipocyte hyperplasia ${ }^{32}$ whereas animal models with reduced adipocyte cell size and increased hyperplasia show improved insulin sensitivity and reduced adipose tissue inflammation ${ }^{48,49}$. Similar correlations between adipogenesis, adipocyte cell size, and metabolic consequences have been observed in humans ${ }^{30,31}$. Although the precise mechanisms linking adipocyte hypertrophy to inflammation and insulin resistance are incompletely understood, hypoxia in the hypertrophic adipose tissue has been shown to be an important mediator of hypertrophy-induced inflammation and insulin resistance ${ }^{50-53}$.

In this study, we show that the mechanosensitive cation Piezo1 on mature adipocytes is a critical mediator of hypercaloric diet induced adipocyte hyperplasia. This discovery identifies a mechanism that is intrinsic to the WAT and increases adipocyte cell number in response to a positive energy balance. Our findings strongly support the concept that mechanical force is a major determinant of obesity and may help to identify novel therapeutic approaches to prevent or treat pathological obesity and associated disorders.

\section{Methods}

Cells. For the isolation of human and mouse adipocyte as well as SVF, adipose tissue was digested with collagenase II $(0.25 \mathrm{mg} / \mathrm{ml}$, Sigma $)$ in a buffer containing $125 \mathrm{mM} \mathrm{NaCl}, 5 \mathrm{mM} \mathrm{KCl}, 1 \mathrm{mM} \mathrm{CaCl} 2,2.5 \mathrm{mM} \mathrm{MgCl}_{2}, 1 \mathrm{mM} \mathrm{KH}_{2} \mathrm{PO}_{4}, 2 \% \mathrm{BSA}$, $4 \mathrm{mM}$ glucose, and $25 \mathrm{mM}$ Tris ( $\mathrm{pH}$ 7.4) for 1 hour at $37^{\circ} \mathrm{C}$. Cells were filtered through a nylon mesh, and the cell suspension including adipocytes and the SVF was washed 3 times with the same buffer while keeping the floating adipocytes untouched. Thereafter, adipocytes were harvested. The wash buffer was filtered through a $40 \mu \mathrm{m}$ filter and was centrifuged for $30 \mathrm{~min}$ at $350 \times g$ to collect the SVF. Isolated adipocytes and SVF were cultured at $37^{\circ} \mathrm{C}$ and $5 \% \mathrm{CO}_{2}$ in DMEM with $10 \% \mathrm{FBS}$ and $100 \mathrm{U} / \mathrm{ml}$ penicillin and streptomycin each.

To prepare conditioned medium from primary adipocytes for SVF and 3T3F442A in vitro differentiation assays, the adipocyte supernatant was collected after $16 \mathrm{~h}$ of culture, centrifuged and then filtered through a $0.22 \mu \mathrm{m}$ filter (Millipore). As a control for experiments using the conditioned medium, SVF cells was either kept in non-conditioned medium or were kept 2 days after reaching confluency in DMEM containing $1 \mu \mathrm{g} / \mathrm{ml}$ insulin (Gibco), $0.25 \mu \mathrm{g} / \mathrm{ml}$ dexamethasone (Sigma), $30 \mu \mathrm{g} / \mathrm{ml} \mathrm{3-isobutyl-1-methylxanthine} \mathrm{(Sigma)} \mathrm{to} \mathrm{induce}$ differentiation of adipocyte precursor cells. Conditioned medium was given to the SVF 2 and 4 days after cells had reached confluency. Thereafter cells were cultured for an additional 6 days in DMEM supplemented $5 \mu \mathrm{g} / \mathrm{ml}$ insulin with medium changes every 2 days. 3T3-F442A cells were obtained from the European Collection of Authenticated Cell Cultures (ECACC), and cells were grown in DMEM with $10 \% \mathrm{FBS}$ and $100 \mathrm{U} / \mathrm{ml}$ penicillin and streptomycin each. As a control for experiments using conditioned medium, 3T3-F442A cells were kept in the nonconditioned medium. To induce differentiation, 3T3-F442A cells were cultured in
DMEM plus $10 \%$ FBS and $5 \mu \mathrm{g} / \mathrm{ml}$ insulin after reaching confluency for 2 days. Conditioned medium was given to 3T3-F442A cells 2 and 4 days after cells had reached confluency. Thereafter cells were cultured for 4 days in DMEM with $10 \%$ FBS. In some experiments, the SVF was incubated with 10 nM FGF1 (R\&D) without or with $10 \mu \mathrm{M}$ LY294002 (Sigma), $10 \mu \mathrm{M}$ SB253580 (Enzo Life Science) or $10 \mu \mathrm{M}$ PD98059 (Sigma) for 2 days, and the expression of adipocyte marker genes Ppary 2 and C/ebpa was determined.

To prepare conditioned medium for adipogenic mediator and lipid measurment, freshly isolated primary adipocytes were washed and then cultured for $16 \mathrm{~h}$ in DMEM with $10 \%$ FBS and $100 \mathrm{U} / \mathrm{ml}$ penicillin and streptomycin. This basal conditioned medium was harvested, centrifuged at $1000 \times g$ for $10 \mathrm{~min}$ at $4{ }^{\circ} \mathrm{C}$ to remove any cell debris, and filtered through a $0.22 \mu \mathrm{m}$ filter (Millipore) for Elisa and LC-MS/MS analysis. To immunodeplete FGF1 from adipocyte-conditioned medium, $2 \mu \mathrm{g}$ of monoclonal anti-FGF1 antibody (Santa Cruz, \# sc-55520) or control IgG (Santa Cruz, \# sc-3879) were added to $200-300 \mu$ l of adipocyteconditioned medium filtrates and incubated for $15 \mathrm{~h}$ at $4{ }^{\circ} \mathrm{C}$ under gentle agitation. $60 \mu \mathrm{l}$ of protein $\mathrm{G}$-coupled agarose beads (Santa Cruz) were added to the solution and gently mixed, followed by incubation for $6 \mathrm{~h}$ at $4{ }^{\circ} \mathrm{C}$ under gentle agitation. Supernatants were collected and filtered after centrifugation at $300 \times g$ for $30 \mathrm{~s}$ and used for differentiation experiments ${ }^{54}$.

To measure Yodal induced release of adipogenic mediators and lipids, freshly isolated primary adipocyte were cultured for $16 \mathrm{~h}$ in DMEM with $10 \%$ FBS and 100 $\mathrm{U} / \mathrm{ml}$ penicillin and streptomycin. Cells were then gently washed 5 times with HBSS supplemented with $2 \%$ fatty acid-free BSA. Thereafter, the medium was replaced by fresh HBSS containing Yodal or carrier solution ( $0.1 \%$ DMSO) for the indicated time periods. This medium was then harvested, centrifuged, and filtered for Elisa and LC-MS/MS analysis as described above. To induce hypotonic membrane stress, primary adipocytes were exposed to hypotonic buffer contained (in $\mathrm{mM}$ ): $90 \mathrm{NaCl}, 2 \mathrm{CsCl}, 1 \mathrm{MgCl}_{2}, 1 \mathrm{CaCl}_{2}, 10$ HEPES, 10 mannitol, pH 7.4 with $\mathrm{NaOH}$ $\left(210 \mathrm{mOsm} \mathrm{kg}^{-1}\right)$. Isotonic extracellular solution had the same composition, but contained $110 \mathrm{mM}$ instead of $10 \mathrm{mM}$ mannitol $\left(300 \mathrm{mOsm} \mathrm{kg}{ }^{-1}\right)^{46}$.

Oil-Red-O and BODIPY staining. Differentiated SVF and 3T3-F442A cells were fixed with $2 \%$ formaldehyde and $0.2 \%$ glutaraldehyde in PBS for $15 \mathrm{~min}$ and then washed 3 times in PBS for 10 min. For Oil-Red-O staining, cells were washed for $30 \mathrm{~s}$ in $60 \%$ isopropanol and stained with Oil-Red-O $(0.7 \%$ in $60 \%$ isopropanol; Sigma) for $1 \mathrm{~h}$ and rinsed with $60 \%$ isopropanol for $1 \mathrm{~min}$ followed by water. OilRed-O stained cells were directly imaged using an inverted microscope or lipid content was quantified after Oil-Red-O extraction with isopropanol by determination of light absorbance at $490 \mathrm{~nm}$. For BODIPY staining, fixed cells were blocked in 2\% BSA in PBS with $0.3 \%$ Triton X-100 for $1 \mathrm{~h}$ and incubated with BODIPY (Invitrogen, catalog number D3922, 1:500), DAPI (Invitrogen, catalog number D1306, 1:1000) and anti-Ki67 antibodies (Abcam, catalog number ab15580, 1:200) for $1 \mathrm{~h}$ at room temperature. Cells were washed and incubated with donkey anti-rabbit AF-594 (Molecular Probes \#A32754, 1:500) secondary antibody for $1 \mathrm{~h}$ and washed with PBS, followed by imaging with Leica TCS SP5 confocal microscope.

siRNA-mediated knock-down. At density of $1-2 \times 10^{4} / \mathrm{cm}^{2}$, cells were transfected with siRNAs using Opti-MEM and Lipofectamine RNAiMAX (Invitrogen) ${ }^{55}$. SiRNAs were from Qiagen or Sigma. 50 pmoles siRNA were mixed gently with RNAiMAX in $100 \mu \mathrm{l} \mathrm{Opti-MEM}$ and incubated for $30 \mathrm{~min}$ at room temperature and added to $1.5 \mathrm{ml}$ cell culture medium (DMEM). Cells were incubated with the complexes for $6 \mathrm{~h}$ at $37^{\circ} \mathrm{C}$ in a $\mathrm{CO}_{2}$ incubator and thereafter medium was replaced by complete medium (DMEM plus $10 \%$ FBS). The transfection was repeated next day and assays were performed $48 \mathrm{~h}$ after the second transfection. The targeted sequences of siRNAs directed against RNAs encoding PIEZO1 were 5'-CACCGGCATCTACGTCAAATA$3^{\prime}$ and $5^{\prime}$-TCGGCGCTTGCTAGAACTTCA- $3^{\prime}$. 
Determination of membrane tension in primary adipocytes. Primary visceral adipocytes from mice fed with standard diet or HFD for 7 days were isolated and incubated with $1 \mu \mathrm{M}$ of the membrane tension probe Flipper-TR ${ }^{\circledast}$ (Tebu-bio, \#SC020) for $30 \mathrm{~min}$ at $37^{\circ} \mathrm{C}$ as described ${ }^{29}$. The adipocytes were then washed 3 times with HBSS and imaged with a Leica-SP8 FLIM microscope. Excitation was performed using a pulsed $488 \mathrm{~nm}$ laser (Laser kit WLL2+pulse picker, Leica Microsystems) operating at $80 \mathrm{MHz}$, and the emission signal was collected from 549 to $651 \mathrm{~nm}$ with acousto-optical beam splitter (AOBS) using a gated hybrid (HyD SMD) detectors and a TimeHarp 300 TCSPC Module and Picosecond Event Timer (PicoQuant). SymPhoTime 64 software (PicoQuant) was then used to fit fluorescence decay data. To extract lifetime information, the photon histograms from membrane regions were fitted with a double exponential, and 2 fluorescence emission decay times ( $\tau 1$ and $\tau 2)$ are extracted. The longest lifetime with the higher fit amplitude $\tau 1$ is used to report membrane tension ${ }^{29}$.

Determination of $\left[\mathrm{Ca}^{2+}\right]_{\mathrm{i}}$. For the determination of the intracellular $\mathrm{Ca}^{2+}$ concentration, 3T3-F442A cells and adipocytes were loaded with $5 \mu \mathrm{M}$ Fluo-4 AM (Molecular Probes, \#F14201) for $1 \mathrm{~h}$ and attached with $0.025 \%$ polyethyleneimine (Sigma) for $30 \mathrm{~min}$ on a glass imaging plate from Eppendorf. Live-cell images were acquired with an Olympus IX81 microscope. Fluorescent intensity was measured with a FlexStation 3 (Molecular Devices).

Electrophysiology. Cell attached patch-clamp recordings (borosilicate pipette resistance of 1.2-1.4 Mohms) were performed as previously described ${ }^{56,57}$. Briefly, currents were elicited by a HSPC1 high speed pressure clamp (Ala, USA) at various holding potentials, as indicated. The pipette solution was: $150 \mathrm{NaCl}, 5 \mathrm{KCl}, 1 \mathrm{Mg}^{2+}$, $2 \mathrm{Ca}^{2+}, 10$ Hepes, $\mathrm{pH}$ : 7.35. The bath solution contained: $150 \mathrm{KCl}, 3 \mathrm{Mg}^{2+}$, 10 Hepes, 5 EGTA, pH: 7.2. Currents were recorded with a Multiclamp 700B patch clamp amplifer (Axon Instruments, USA), filtered at $1 \mathrm{kHz}$ and digitized at $10 \mathrm{kHz}$ using Clampex (Axon Instruments, USA).

Immunoblotting. Cells and tissues were lysed in RIPA buffer (Cell Signaling Technology, \#9806) supplemented with protease and phosphatase inhibitors (Cell Signaling Technology, \#5872). Lysates were centrifuged at $10,000 \times g$ for $10 \mathrm{~min}$ then subjected to SDS-PAGE and transferred to nitrocellulose membranes. Membranes were probed with primary and HRP-conjugated secondary antibodies (Cell Signaling Technology) and were developed using the ECL detection system (Pierce). Anti-Piezo1-antibody was from Proteintech (catalog number 15939-1-AP, 1:200), anti-GAPDH, anti-FGFR1 and anti-AKT antibodies were from Cell Signaling Technology (\#2188,\#9740 and \#9272, respectively, 1:500) and anti-FGF1 antibody was from Santa Cruz Biotechnology (sc-55520, 1:200).

Animal models. All mice were back-crossed onto a C57BL/6N background at least 8-10 times, and experiments were performed with littermates as controls. For in vivo experiments, male animals were used at an age of 8-12 weeks if not stated otherwise, while adipose tissue from female and male mice were tested in parallel in several in vitro experiments as indicated. Mice were housed under a 12-h light-dark cycle with free access to food and water and under specific pathogenfree conditions if not stated otherwise. Mice carrying a floxed allele of the gene encoding Piezo ${ }^{26}$ and Fgfr ${ }^{34}$ as well as the Cre reporter line Gt(ROSA)26Sor ${ }^{\mathrm{tm}} 4$ (ACTB-tdTomato,-EGFP)Luo/J ( $\mathrm{mT} / \mathrm{mG})^{58}$ were obtained from The Jackson Laboratory. Animals expressing the lacZ gene under the control of the Piezol promoter were obtained from the Knockout Mouse Project (KOMP). Mice that allow for tamoxifen-dependent adipocyte-specific Cre activation have been described before $^{59}$ and Pdgfra-CreERT2 animals ${ }^{35}$ were provided by the RIKEN BRC through the National BioResource Project of the MEXT/AMED, Japan (RBRC09616). To induce recombination, animals were treated with $5 \times 1 \mathrm{mg} / \mathrm{d}$ tamoxifen (Sigma) dissolved in corn oil, and 7-14 days later experiments were started. All animals which served as controls for tamoxifen-induced adipocytespecific animals were treated with the same amount of tamoxifen under the same conditions. Mice were fed a standard pellet chow (Altromin) or a HFD containing $30 \%(w / w)$ fat (ssniff) with water ad libitum.

Calorimetry. Mice were placed into the analysis cages of the PhenoMaster-System (TSE-Systems, Bad Homburg, Germany) for $48 \mathrm{~h}$. Following $48 \mathrm{~h}$ of acclimatization, $\mathrm{O}_{2}$ consumption and $\mathrm{CO}_{2}$ production were measured every $10 \mathrm{~min}$ for a total of $48 \mathrm{~h}$ and converted to $\mathrm{kcal} / \mathrm{h}$ using the Weir equation. Data were analyzed using the PhenoMaster-Software (TSE-Systems, Bad Homburg, Germany).

Glucose and insulin tolerance test. After overnight fasting glucose was administered to mice intraperitoneally at a dose of $2 \mathrm{mg} / \mathrm{g}$ body weight when on SD and of $2.75 \mathrm{mg} / \mathrm{g}$ body weight when on HFD. Blood samples were taken from the tail vein before and after glucose application at indicated time points, and glucose levels were measured using a glucometer (Accu-Chek Aviva). For insulin tolerance test, mice were injected intraperitoneally with insulin $(0.75 \mathrm{mU} / \mathrm{g})$, and glucose levels were determined as described above.
Histology, immunohistochemistry, TUNEL assay. H\&E staining was performed on 4- $\mu \mathrm{m}$ paraffin sections according to standard protocols. For immunohistochemical staining of adipose tissue sections, $12-\mu \mathrm{m}$ cryosections were treated according to the instructions of the In Situ Cell Death Detection Kit (Roche Applied Sciences). After washing twice in PBS, slides incubated for 10 min with DAPI (Invitrogen), and finally mounted in Mowiol (Calbiochem/EMD Biosciences) and analyzed on a Leica TCS SP5 confocal microscope by an experimenter blinded to genotype. Using the ImageJ software adipocyte volume was calculated from $\mathrm{H} \& \mathrm{E}$ stained paraffin sections as describes previously ${ }^{60}$. Adipocyte number was calculated by dividing epididymal adipose tissue volume, calculated by multiplying epididymal adipose tissue weight and commonly used adipose tissue density factor $0.92 \mathrm{~g} / \mathrm{cm}^{3}$, by adipocyte volume ${ }^{61}$.

BrdU and EdU labeling. For BrdU in vivo proliferation assay, mice were supplied with water containing $1 \mathrm{mg} / \mathrm{ml} \mathrm{BrdU}$ (Merck; catalog number 203806) for 1 week. In parallel, feeding of mice with HFD was initiated. After 1 or 8 weeks, adipose tissue was prepared, minced, and fixed in zinc formaldehyde for $24 \mathrm{~h}$ at $4{ }^{\circ} \mathrm{C}$. The samples were then washed and blocked with 2\% BSA in PBS and stained overnight at $4{ }^{\circ} \mathrm{C}$ with antibodies including anti-Perilipin-1 (Abcam \# ab61682, 1:500), antiBrdU (Abcam \# ab6326, 1:300), anti-PDGFRa (R\&D \#AF1062, 1:200). The samples were then washed with PBS three times for $10 \mathrm{~min}$ at room temperature. Secondary antibodies including goat anti-mouse AF-546, (Molecular Probes \# A11003, 1:500), donkey anti-rabbit AF-488 (Molecular Probes \#A11008, 1:500), rabbit anti-goat AF-594 (Molecular Probes \#A11080, 1:500) were applied in PBS with $2 \%$ BSA and $0.3 \%$ Triton X-100. Some tissues were further incubated with DAPI (Invitrogen), LipidTOX (Molecular Probes \#H34475) for neutral lipid staining and CellMask Orange (Molecular Probes \#C10045, 1:400) for plasma membrane stain for $1 \mathrm{~h}$ at room temperature, followed by three washes with PBS at room temperature in the dark. The tissues were then imaged with a Leica confocal SP5 microscope. Adipocyte nuclei were identified by their location between plasma membrane and adipocyte lipid droplets as described by Jeffery et al. ${ }^{15}$. Nuclei that are located between two plasma membranes are non-adipocyte nuclei and nuclei that are not separated from the lipid droplets by a plasma membrane are the mature adipocyte nuclei.

For EdU in vivo incorporation assay, mice were supplied with water containing $500 \mu \mathrm{g} / \mathrm{ml}$ EdU (Lumiprobe; catalog number 40540) for 1 week. In parallel, feeding of mice with HFD was initiated. After 1 or 8 weeks, adipocytes and SVF from epididymal WAT were isolated as described above.

Caspase-3 activity assay. White adipose tissue or isolated adipocytes were homogenized in a dounce homogenizer in lysis buffer $(10 \mathrm{mM}$ Tris- $\mathrm{HCl}, 10 \mathrm{mM}$ $\mathrm{Na}_{2} \mathrm{HPO}_{4} / \mathrm{NaH}_{2} \mathrm{PO}_{4}, \mathrm{pH} 7.5,130 \mathrm{mM} \mathrm{NaCl}, 1 \%$ Triton -X-100, $\left.10 \mathrm{mM} \mathrm{NaPPi}\right)$ and kept on ice for $30 \mathrm{~min}$. Samples were centrifuged for $15 \mathrm{~min}$ at $4{ }^{\circ} \mathrm{C}$ and $18.400 \times g$, and supernatants taken for caspase- 3 activity assay and protein concentration determination. For caspase-3-activity assays $20 \mu \mathrm{l}$ sample and $200 \mu \mathrm{l}$ assay buffer (20 mM HEPES ( $\mathrm{pH} 7.5$ ), 10\% glycerol, $2 \mathrm{mM}$ DTT, $20 \mu \mathrm{M} \mathrm{Ac-}$ DEVD-AMC) were incubated at $37^{\circ} \mathrm{C}$ for $2 \mathrm{~h}$ and luminescence was measured (FlexStation 3, Molecular Devices).

Adipocyte pulse-chase experiments. For adipocyte pulse-chase experiments, mice were treated with $5 \times 1 \mathrm{mg} / \mathrm{d}$ tamoxifen by intraperitoneal injection for 5 consecutive days starting at 8 weeks of age, and were then allowed to recover for 1 week. Mice were then placed on HFD or remained on SD for a 1- or 8-week chase period. Inguinal and epididymal WAT were taken from several regions throughou the depot, and analyzed by whole-mount confocal microscopy for tdTomato and eGFP expression. For each data point, at least 300 (sWAT) or 2000 (vWAT) adipocytes were counted from multiple images from each depot of each animal.

Flow cytometry and cell sorting. For adipocyte sorting and size distribution analysis, primary mouse adipocytes were freshly isolated from inguinal or epididymal fat pads as described above and then stained with Hoechst 34580 (392/ $440 \mathrm{~nm}$, Invitrogen \#H21486, 1:500) and Calcien-AM (495/515 nm, BD \#564061, 1:500). In experiments involving $\mathrm{mT} / \mathrm{mG}$ reporter mice, adipocytes were only stained with Hoechst. The cell suspension was then loaded into the sample cartridge and analyzed or sorted using a BioSorter large particle flow cytometer (Union Biometrica) equipped with a $500 \mu \mathrm{m}$ metal-free FOCA (Fluidics and Optics Core Assembly). Physical characteristics of size (TOF), optical density (EXT), and three different channels of fluorescence signals (FLU) were collected. Adipocytes were carried through the flow cell, one by one, and passed through the focus of a laser beam. Relative size was determined by the time of flight (TOF) measurement and calibrated with megabead particle size standards (Polysciences). The optical density of the object was determined by the extinction (EXT) measurement. Spatial information on fluorescence and extinction for each adipocyte passing through the flow cell were collected by using Profiler $\mathrm{II}^{\mathrm{mw}}$. Solid state $405 \mathrm{~nm}$ and $488 \mathrm{~nm}$ lasers were used in the experiments. Calcein-AM or GFP fluorescence was collected using BP510/23 nm filter (PMT2) and Hoechst fluorescence using BP 440/30 nm (PMT1).

For analysis of EdU incorporation, adipocyte nuclei was prepared by lysing adipocytes for $5 \mathrm{~min}$ in $0.2 \%$ IGEPAL in HBSS with vortexing every $1-2 \mathrm{~min}$ and 
centrifugation at $3000 \times g$ for $10 \mathrm{~min}$. SVF was first stained with extracellular antibodies including anti-PDGFRa (APC, eBioscience \#17-1401), anti-CD34 (AF700, eBioscience \#57-0341), anti-CD31 (efluor450, eBioscience \#48-0311-80) and anti-CD45 (efluor450, eBioscience \#48-0451-80, 1:100) antibodies for $30 \mathrm{~min}$ on ice, and then washed with PBS. Both adipocyte nuclei and SVF were fixed in Fix/Perm (eBioscience \#00-512343) and permeabilized with permeabilization buffer (eBioscience \#00833356). Samples were then treated with $6000 \mathrm{unit} / \mathrm{ml}$ DNase (Fisher Scientific) in PBS for $2 \mathrm{~h}$ and processed for propidium Iodide (Invitrogen R37169) staining and EdU staining using Edu Alexa Fluor ${ }^{\text {mix }} 488$ or 647 Flow Cytometry Assay Kit (Invitrogen \#C10632 and \#C10634). Flow cytometry data were collected on BD FACS Canto II (Becton Dickinson) and analyzed using the FlowJo software package. For assessment of lipid positive cell numbers after SVF differentiation, SVF cells differentiated in 6-well plate were trypsinized, fixed in Fix/Perm (eBioscience \#00-512343) and permeabilized with permeabilization buffer (eBioscience \#00833356). Half of cells were then stained for BODIPY (Invitrogen, catalog number D3922). BODIPY was excited with a $488 \mathrm{~nm}$ green laser and emission was recorded at $503 \mathrm{~nm}$ using a BD FACS Canto II (Becton Dickinson). The rest of the cells were stained for Oil Red O as described above to calculate lipid content.

Plasma levels of insulin and IL-6. Blood samples were collected from the tail vein or the retro-orbital plexus in EDTA (10 mM final concentration). Plasma IL-6 and insulin levels were determined using the MAGPIX system combined with MILLIPLEX MAP magnetic bead-based multi-analyte panels and MILLIPLEX Analyst 5.1 software (Merck Millipore).

Determination of adipogenic mediators and lipids. Concentrations of IGF-1, FGF-1, FGF-2, Jag-1, TGF- $\beta$, BMP3, adiponectin, WNT-5a and WNT10B in supernatants of adipocytes were determined using ELISA kits. IGF-1 and TGF- $\beta$ ELISAs were from Bio-Techne GmbH. FGF-1 and BMP2 ELISA was from Abcam, adiponectin ELISA kit was from Millipore and FGF-2, Jag-1, BMP3, WNT-5a, and WNT10B ELISAs were from antibodies-online GmbH.

For lipid measurement, conditioned medium $(500 \mu \mathrm{l})$ was spiked with $10 \mu \mathrm{l}$ internal standard stock solution which containing 22 deuterium labeled oxylipin standards and ${ }^{13} \mathrm{C}$-labeled arachidonic acid. Lipids were extracted using a twophase liquid-liquid extraction in which ethyl acetate $(1000 \mu \mathrm{l})$ was added, samples were rigorous vortexed, centrifuged $\left(10,000 \times g, 5 \mathrm{~min}, 4^{\circ} \mathrm{C}\right)$, and the upper organic phase was collected. The extraction was repeated one more time. These lipid extracts were evaporated to dryness under a continuous nitrogen stream (Vacuum manifold, Macherey-Nagel, Düren, Germany) and subsequently resuspended in methanol/water (1:1). $8 \mu \mathrm{l}$ of each sample were analyzed by UPLCMS/MS for primary fatty acids and oxylipins. The polyunsaturated fatty acid (PUFA)-derived lipid mediators were detected using a custom LC-MS/MS screening library that was developed to assess the involvement of such mediators in inflammation, angiogenesis, and metabolic diseases. The assay used detects 112 unique lipid species, derived from cyclooxygenase, lipoxygenase, and cytochrome P450 metabolism. The UPLC-MS/MS analyses were performed as described (11), with minor modifications. Reversed-phase separation was performed on an Acquity UPLC BEH shield RP18 column $(2.1 \times 100 \mathrm{~mm} ; 1.7 \mu \mathrm{m}$; Waters, Milford, USA $)$ on an Agilent 1290 Infinity LC5 system (Agilent, Waldbronn, Germany). The mobile phase consisted of (A) ACN/water/acetic acid (60/40/0.02, v/v/v) and (B) ACN/ isopropanol $(50 / 50, \mathrm{v} / \mathrm{v})$. Elution of analytes was carried out for $5.8 \mathrm{~min}$ at a flow rate of $0.5 \mathrm{~mL} / \mathrm{min}$. Gradient conditions were as follows: $0-4.5 \mathrm{~min}, 0.1-55 \% \mathrm{~B}$; 4.5-5.0 min, 55-99\% B; 5.0-5.8 min, 99\% B, followed by are-equilibration step $0.1 \%$ B for 2 min. Mass spectrometry was performed on a QTrap 5500 mass spectrometer (Sciex, Darmstadt, Germany), equipped with a Turbo V ion source. Electro spray ionization in negative mode was employed. The ion source parameter were as followed, CUR $20 \mathrm{psi}$, IS $-4500 \mathrm{~V}$, TEM $525^{\circ} \mathrm{C}$, GS1 $30 \mathrm{psi}$, GS2 $30 \mathrm{psi}$, $\mathrm{CAD}$ medium (nitrogen was employed as the collision gas). Analyst 1.6.2 and MultiQuant 3.0 (Sciex, Darmstadt, Germany), were used for data acquisition and analysis, respectively.

Expression analysis. RNA isolation and transcription was performed as described previously ${ }^{55}$. Total RNA was isolated from cells using the RNeasy mini or micro kit (Qiagen) according to the manufacturer's protocol. For fat tissues, RNeasy lipid tissue Mini Kit (Qiagen) was used to isolate total RNA. DNA was removed using the QIAGEN RNase-Free DNaseSet. Complementary DNA synthesis was performed using the ProtoScript II Reverse Transcription kit (New England BioLabs, M0368S). Primers were designed with the online tool provided by Roche and quantification was performed using the LightCycler 480 Probe Master System (Roche). Genomic DNA was used as a universal standard to calculate gene copy number per nanogram of RNA. Relative expression levels were obtained by normalization with GAPDH or $18 \mathrm{~S}$ values.

qPCR primer sequences. CD11b $5^{\prime}$-tctggcagatgtggctattg- $3^{\prime}$ and $5^{\prime}$ gtcctgtcttgaggctccat- $3^{\prime}$

CD68 $5^{\prime}$-ttctgctgtggaaatgcaag- $3^{\prime}$ and $5^{\prime}$-agagagagcaggtcaaggtga- $3^{\prime}$;

Nos2 $5^{\prime}$-aaggggacgaactcagtgg- $3^{\prime}$ and $5^{\prime}$-cccggaaggtttgtacagc- $3^{\prime}$

Arg1 $5^{\prime}$-ggcctttgttgatgtcccta- $3^{\prime}$ and $5^{\prime}$-acagaccotgggttcttcac- $3^{\prime}$;
CD137 $5^{\prime}$-caagagctgccctccaagta- $3^{\prime}$ and $5^{\prime}$-cagatgttacagttcggctgtc- $3^{\prime}$ CD11c $5^{\prime}$-gagccagaacttcccaactg and $5^{\prime}$-tcaggaacacgatgtcttgg- $3^{\prime}$;

HOXC1 $5^{\prime}$-gcagcaagcacaaagagga- $3^{\prime}$ and $5^{\prime}$-cgtctggtacttggtgtaggg- $3^{\prime}$ $\mathrm{F} 4 / 805^{\prime}$-tctctaaactcaaggacacgaggt- $3^{\prime}$ and $5^{\prime}$-ctggaaaatgcccagcac- $3^{\prime}$; IL6 $5^{\prime}$-tgatggatgctaccaaactgg- $3^{\prime}$ and $5^{\prime}$-ttcatgtactccaggtagctatg- $3^{\prime}$;

IL1A $5^{\prime}$-ttggttaaatgacctgcaaca- $3^{\prime}$ and $5^{\prime}$-gagcgctcacgaacagttg- $3^{\prime}$;

MRC2 $5^{\prime}$-ccccaaactccgacactg- $3^{\prime}$ and $5^{\prime}$-gggcctggatccaactct- $3^{\prime}$;

TNF $\alpha 5^{\prime}$-tcttctcattcctgcttgtgg- $3^{\prime}$ and $5^{\prime}$-ggtctgggccatagaactga- $3^{\prime}$;

Adipoq $5^{\prime}$-cctaccttccttccagactgtgt- $3^{\prime}$ and $5^{\prime}$-agaagagaaagaaacccagcaa- $3^{\prime}$; Perilipin $5^{\prime}$-ggacttacaaacagcaacagacc- $3^{\prime}$ and $5^{\prime}$-catctgtgagttggtggacact- $3^{\prime}$; PPAR $\gamma 15^{\prime}$-acgcgaggaggtcaagaag- $3^{\prime}$ and $5^{\prime}$-tcaatgggagttaagaagaattt- $3^{\prime}$; PPAR $\gamma^{2} 5^{\prime}$-tctgtgtcaaccatggtaatttc- $3^{\prime}$ and $5^{\prime}$-tgctgttatgggtgaaactctg- $3^{\prime}$; Cebpa $5^{\prime}$-cgctggtgatcaaacaagag- $3^{\prime}$ and $5^{\prime}$-ggtggctggtaggggaag- $3^{\prime}$; FABP4 $5^{\prime}$-caggctcagactcagcagttt- $3^{\prime}$ and $5^{\prime}$-cacagggaactgagcagtga- $3^{\prime}$; FAS $5^{\prime}$-gcaggcagtcagtcagca- $3^{\prime}$ and $5^{\prime}$-aggctttctcccttagggtct- $3^{\prime}$;

CD36 $5^{\prime}$-ttgaaaagtctcggacattgag- $3^{\prime}$ and $5^{\prime}$-tcagatccgaacacagcgta- $3^{\prime}$; FGFR1 $5^{\prime}$-ttgaaagattatgttttggttctctg- $3^{\prime}$ and $5^{\prime}$-tggtgaactgtcttgtatccttaaac- $3^{\prime}$; FGFR2 $5^{\prime}$-tgatagcagggtcctctcgt- $3^{\prime}$ and $5^{\prime}$-caaggcaaacgtgatgaatg- $3^{\prime}$; FGFR3 $5^{\prime}$-ctgagaacgtgcgtctgg- $3^{\prime}$ and $5^{\prime}$-tgactaggagagccattcaagc- $3^{\prime}$; FGFR4 $5^{\prime}$-cctgagatcagctggaagga- $3^{\prime}$ and $5^{\prime}$-tcccctgaaagatgctcaac- $3^{\prime}$; FGF1 $5^{\prime}$-cctgccagttcttcagtgc and $5^{\prime}$-ggctgcgaaggttgtgat- $3^{\prime}$;

Piezo1 (human) $5^{\prime}$-cgtcttcgtggagcagatg and $5^{\prime}$-gcccttgacggtgcatac- $3^{\prime}$;

Piezo2 (human) $5^{\prime}$-cactcagaaaatgcaacagca- $3^{\prime}$ and $5^{\prime}$-ttccctctcttttgctcctaga- $3^{\prime}$;

Piezo1 (mouse) $5^{\prime}$-ggaaaagagctccgacacac- $3^{\prime}$ and $5^{\prime}$-ccaggacttccccacctatt- $3^{\prime}$;

Piezo2 (mouse) $5^{\prime}$-agatcaagatgggcaacagg- $3^{\prime}$ and $5^{\prime}$-ttcgtgattatcctgaaccaca- 3

Other materials. Insulin was from Gibco. Yodal was from Maybridge (catalog number SPB07298). BAPTA-AM (catalog number B1205) was from Invitrogen. PD173074 (catalog number P2499) and brefeldin A (catalog number B5936) were from Sigma. Recombinant FGF1 was from R\&D (catalog number 4686-FA). AntiCD68 antibody was from Bio-Rad (catalog number MCA1957).

Statistics. Trial experiments or experiments done previously were used to determine sample size with adequate statistical power. Samples were excluded in cases where RNA/cDNA quality or tissue quality after processing was poor (below commonly accepted standards). Animals were excluded from experiments if they showed any signs of sickness. The investigator was blinded to the group allocation and during the experiment. Data represent biological replicates. In all studies, comparison of mean values was conducted with unpaired, two-tailed Student's ttest or one-way or two-way ANOVA with Bonferroni's post hoc test. In all analyses, statistical significance was determined at the $5 \%$ level $(P<0.05)$. Depicted are mean values \pm s.d. or \pm s.e.m. as indicated in the figure legends. Statistical analysis was performed with Prism5 or Prism6 (GraphPad) or Excel (Microsoft) software.

Study approval. The work on human adipocyte samples was approved by the ethical committee of Xi' an Jiaotong University (XJTU2018-249 and XJTU2019-12) and conforms to the guidelines of the 2000 Helsinki declaration. Written informed consent was obtained from all subjects before their participation. All procedures of animal care and use in this study were approved by the local animal welfare authorities and committees (Regierungspräsidium Darmstadt, Germany and Ethical Committee of Xi'an Jiaotong University, China).

Reporting summary. Further information on research design is available in the Nature Research Reporting Summary linked to this article.

\section{Data availability}

The source data underlying Figs. 1b, d-f, 2a, b,d-l, 3a-c and e, 4b, c, e-h, 5a-c, 6a-g and i-k and Supplementary Figs. 1a, e-l, 2a-f, 3a, c, e-i, 4a-g are provided as Source Data file. All data are available from the corresponding authors upon request.

Received: 16 October 2019; Accepted: 1 April 2020;

Published online: 08 May 2020

\section{References}

1. Heymsfield, S. B. \& Wadden, T. A. Mechanisms, pathophysiology, and management of obesity. N. Engl. J. Med. 376, 254-266 (2017).

2. Czech, M. P. Insulin action and resistance in obesity and type 2 diabetes. Nat Med. 23, 804-814 (2017).

3. Bluher, M. Obesity: global epidemiology and pathogenesis. Nat. Rev. Endocrinol. 15, 288-298 (2019).

4. Gonzalez-Muniesa, P. et al. Obesity. Nat. Rev. Dis. Prim. 3, 17034 (2017).

5. Berry, R., Jeffery, E. \& Rodeheffer, M. S. Weighing in on adipocyte precursors Cell Metab. 19, 8-20 (2014). 
6. Wang, Q. A., Tao, C., Gupta, R. K. \& Scherer, P. E. Tracking adipogenesis during white adipose tissue development, expansion and regeneration. Nat. Med. 19, 1338-1344 (2013).

7. Ghaben, A. L. \& Scherer, P. E. Adipogenesis and metabolic health. Nat. Rev. Mol. Cell. Biol. 20, 242-258 (2019).

8. Cristancho, A. G. \& Lazar, M. A. Forming functional fat: a growing understanding of adipocyte differentiation. Nat. Rev. Mol. cell Biol. 12, 722-734 (2011).

9. Arner, P. et al. Variations in the size of the major omentum are primarily determined by fat cell number. J. Clin. Endocrinol. Metab. 98, E897-E901 (2013).

10. Spalding, K. L. et al. Dynamics of fat cell turnover in humans. Nature 453, 783-787 (2008).

11. Stenkula, K. G. \& Erlanson-Albertsson, C. Adipose cell size: importance in health and disease. Am. J. Physiol. Regul. Integr. Comp. Physiol. 315, R284-R295 (2018).

12. Rodeheffer, M. S., Birsoy, K. \& Friedman, J. M. Identification of white adipocyte progenitor cells in vivo. Cell 135, 240-249 (2008).

13. Tang, W. et al. White fat progenitor cells reside in the adipose vasculature. Science 322, 583-586 (2008).

14. Vishvanath, L. et al. Pdgfrbeta + mural preadipocytes contribute to adipocyte hyperplasia induced by high-fat-diet feeding and prolonged cold exposure in adult mice. Cell Metab. 23, 350-359 (2016).

15. Jeffery, E., Church, C. D., Holtrup, B., Colman, L. \& Rodeheffer, M. S. Rapid depot-specific activation of adipocyte precursor cells at the onset of obesity. Nat. Cell Biol. 17, 376-385 (2015).

16. Ross, S. E. et al. Inhibition of adipogenesis by Wnt signaling. Science 289, 950-953 (2000).

17. Tang, Q. Q. \& Lane, M. D. Adipogenesis: from stem cell to adipocyte. Annu Rev. Biochem. 81, 715-736 (2012).

18. Rosen, E. D. \& MacDougald, O. A. Adipocyte differentiation from the inside out. Nature reviews. Mol. cell Biol. 7, 885-896 (2006).

19. Coste, B. et al. Piezo proteins are pore-forming subunits of mechanically activated channels. Nature 483, 176-181 (2012).

20. Zhao, Q. et al. Structure and mechanogating mechanism of the Piezol channel. Nature 554, 487-492 (2018).

21. Saotome, K. et al. Structure of the mechanically activated ion channel Piezol. Nature 554, 481-486 (2018).

22. Lewis, A. H. \& Grandl, J. Mechanical sensitivity of Piezol ion channels can be tuned by cellular membrane tension. Elife 4, e12088 (2015).

23. Cox, C. D. et al. Removal of the mechanoprotective influence of the cytoskeleton reveals PIEZO1 is gated by bilayer tension. Nat. Commun. 7, 10366 (2016)

24. Syeda, R. et al. Piezo1 Channels are inherently mechanosensitive. Cell Rep. 17, 1739-1746 (2016).

25. Li, J. et al. Piezol integration of vascular architecture with physiological force. Nature 515, 279-282 (2014).

26. Cahalan, S. M. et al. Piezol links mechanical forces to red blood cell volume. Elife 4, e07370 (2015).

27. Gudipaty, S. A. et al. Mechanical stretch triggers rapid epithelial cell division through Piezol. Nature 543, 118-121 (2017).

28. Coste, B. et al. Piezo1 and Piezo2 are essential components of distinct mechanically activated cation channels. Science 330, 55-60 (2010).

29. Colom, A. et al. A fluorescent membrane tension probe. Nat. Chem. 10, 1118-1125 (2018).

30. McLaughlin, T. et al. Enhanced proportion of small adipose cells in insulinresistant vs insulin-sensitive obese individuals implicates impaired adipogenesis. Diabetologia 50, 1707-1715 (2007).

31. Gustafson, B., Hedjazifar, S., Gogg, S., Hammarstedt, A. \& Smith, U. Insulin resistance and impaired adipogenesis. Trends Endocrinol. Metab. 26, 193-200 (2015).

32. Jonker, J. W. et al. A PPARgamma-FGF1 axis is required for adaptive adipose remodelling and metabolic homeostasis. Nature 485, 391-394 (2012).

33. Prudovsky, I. et al. Folding of fibroblast growth factor 1 Is critical for its nonclassical release. Biochemistry 55, 1159-1167 (2016).

34. Hoch, R. V. \& Soriano, P. Context-specific requirements for Fgfrl signaling through Frs2 and Frs3 during mouse development. Development 133, 663-673 (2006).

35. Miwa, H. \& Era, T. Generation and characterization of PDGFRalphaGFPCreERT2 knock-In mouse line. Genesis 53, 329-336 (2015).

36. Krueger, K. C., Costa, M. J., Du, H. \& Feldman, B. J. Characterization of Cre recombinase activity for in vivo targeting of adipocyte precursor cells. Stem Cell Rep. 3, 1147-1158 (2014).

37. Jeffery, E. et al. Characterization of Cre recombinase models for the study of adipose tissue. Adipocyte 3, 206-211 (2014).

38. Mejhert, N. et al. Mapping of the fibroblast growth factors in human white adipose tissue. J. Clin. Endocrinol. Metab. 95, 2451-2457 (2010).

39. Hutley, L. et al. Fibroblast growth factor 1: a key regulator of human adipogenesis. Diabetes 53, 3097-3106 (2004).
40. He, J. et al. Fibroblast growth factor-1 (FGF-1) promotes adipogenesis by downregulation of carboxypeptidase A4 (CPA4) - a negative regulator of adipogenesis implicated in the modulation of local and systemic insulin sensitivity. Growth Factors 34, 210-216 (2016).

41. Murthy, S. E., Dubin, A. E. \& Patapoutian, A. Piezos thrive under pressure: mechanically activated ion channels in health and disease. Nat. Rev. Mol. Cell Biol. 18, 771-783 (2017).

42. Wu, J., Lewis, A. H. \& Grandl, J. Touch, tension, and transduction-the function and regulation of Piezo ion channels. Trends Biochem Sci. 42, 57-71 (2017).

43. Pope, B. D., Warren, C. R., Parker, K. K. \& Cowan, C. A. Microenvironmental control of adipocyte fate and function. Trends Cell Biol. 26, 745-755 (2016).

44. Shoham, N. \& Gefen, A. Mechanotransduction in adipocytes. J. Biomech. 45 , 1-8 (2012).

45. Del Marmol, J. I., Touhara, K. K., Croft, G. \& MacKinnon, R. Piezo1 forms a slowly-inactivating mechanosensory channel in mouse embryonic stem cells. Elife 7, e33149 (2018)

46. Zhang, Y. et al. SWELL1 is a regulator of adipocyte size, insulin signalling and glucose homeostasis. Nat. Cell Biol. 19, 504-517 (2017).

47. Zhao, C. et al. Mechanosensitive Ion Channel Piezo1 Regulates Diet-Induced Adipose Inflammation and Systemic Insulin Resistance. Front Endocrinol. (Lausanne) 10, 373 (2019).

48. Kim, J. Y. et al. Obesity-associated improvements in metabolic profile through expansion of adipose tissue. J. Clin. Invest 117, 2621-2637 (2007).

49. Senol-Cosar, O. et al. Tenomodulin promotes human adipocyte differentiation and beneficial visceral adipose tissue expansion. Nat. Commun. 7, 10686 (2016)

50. Lee, Y. S. et al. Increased adipocyte O-2 consumption triggers HIF-1 alpha, Causing inflammation and insulin resistance in obesity. Cell 157, 1339-1352 (2014).

51. Reilly, S. M. \& Saltiel, A. R. Adapting to obesity with adipose tissue inflammation. Nat. Rev. Endocrinol. 13, 633-643 (2017).

52. Crewe, C., An, Y. A. \& Scherer, P. E. The ominous triad of adipose tissue dysfunction: inflammation, fibrosis, and impaired angiogenesis. J. Clin. Invest. 127, 74-82 (2017)

53. Sassmann-Schweda, A. et al. Increased apoptosis and browning of TAK1deficient adipocytes protects against obesity. JCI Insight 1, e81175 (2016).

54. Chakraborty, D. et al. Fibroblast growth factor receptor is a mechanistic link between visceral adiposity and cancer. Oncogene 36, 6668-6679 (2017).

55. Wang, S. et al. Endothelial cation channel PIEZO1 controls blood pressure by mediating flow-induced ATP release. J. Clin. Invest 126, 4527-4536 (2016).

56. Retailleau, K. et al. Piezol in smooth muscle cells is involved in hypertensiondependent arterial remodeling. Cell Rep. 13, 1161-1171 (2015).

57. Peyronnet, R. et al. Piezo1-dependent stretch-activated channels are inhibited by Polycystin-2 in renal tubular epithelial cells. EMBO Rep. 14, 1143-1148 (2013).

58. Muzumdar, M. D., Tasic, B., Miyamichi, K., Li, L. \& Luo, L. A global doublefluorescent Cre reporter mouse. Genesis 45, 593-605 (2007).

59. Sassmann, A., Offermanns, S. \& Wettschureck, N. Tamoxifen-inducible Cremediated recombination in adipocytes. Genesis 48, 618-625 (2010).

60. Chen, H. C. \& Farese, R. V. Jr. Determination of adipocyte size by computer image analysis. J. Lipid Res. 43, 986-989 (2002).

61. Bourgeois, F., Alexiu, A. \& Lemonnier, D. Dietary-induced obesity: effect of dietary fats on adipose tissue cellularity in mice. Br. J. Nutr. 49, 17-26 (1983)

\section{Acknowledgements}

The authors wish to thank Svea Hümmer for secretarial help, Wencai Zhao, Martina Finkbeiner, Ulrike Krüger, Claudia Ullmann, Carola Meyer for technical help and Yin Hao for fluorescence-lifetime imaging microscopy. E.H. thanks the Human Frontier Science Program and the ADIPOPIEZO ANR-19-CE14-0029-01 and MECANOADIPO ANR-17-CE13-0012-02 for support. This work was supported by the National Natural Science Foundation of China (grant \#81870220), Shaanxi Natural Science Fund for Distinguished Young Scholars of China (S2020-JC-JQ-0239), the Max Planck Society and the Collaborative Research Centers 834 and 1039 of the German Research Foundation.

\section{Author contributions}

S.P.W. initiated and designed the study, performed experiments, analyzed data and wrote part of the paper; S.C., Y.S., S.K., J.S., and L.W. helped with in vitro and in vivo experiments; M.A. and D.L. performed cell isolation and culture, qPCR experiments and $\mathrm{X}$-gal staining. J.H. and I.F. did the lipidomic analyses; A.A. did cell sorting experiments; Z.W., C.W., and W.Z. did experiments with human adipocytes; N.W. supervised study and discussed data; E.H. initiated and designed the study, performed electrophysiology experiments and discussed data; S.O. designed and supervised the study, discussed data and wrote the paper. All authors commented on the paper.

\section{Competing interests}

The authors declare no competing interests. 


\section{Additional information}

Supplementary information is available for this paper at https://doi.org/10.1038/s41467020-16026-w.

Correspondence and requests for materials should be addressed to S.W. or S.O.

Peer review information Nature Communications thanks the anonymous reviewer(s) for their contribution to the peer review of this work.

Reprints and permission information is available at http://www.nature.com/reprints

Publisher's note Springer Nature remains neutral with regard to jurisdictional claims in published maps and institutional affiliations. (c) (i) Open Access This article is licensed under a Creative Commons Attribution 4.0 International License, which permits use, sharing, adaptation, distribution and reproduction in any medium or format, as long as you give appropriate credit to the original author(s) and the source, provide a link to the Creative Commons license, and indicate if changes were made. The images or other third party material in this article are included in the article's Creative Commons license, unless indicated otherwise in a credit line to the material. If material is not included in the article's Creative Commons license and your intended use is not permitted by statutory regulation or exceeds the permitted use, you will need to obtain permission directly from the copyright holder. To view a copy of this license, visit http://creativecommons.org/ licenses/by/4.0/.

(c) The Author(s) 2020, corrected publication 2022 